wurde wegen des änsserst klaren Fibrillenverlaufs gezeichnet. Der rechts gelegene Theil der Zelle mit dem Kern ist abgeschnitten; die hier vereinzelt und in verschiedenen Richtungen in verlaufenden Fibrillen wurden bei etwas tieferer Einstellung als die übrige Zelle gezeichnet. Im Uebrigen siehe Erklärung zu Fig. 4 und 5.

Fig. 7. Gitterähnliche Anordnung der Primitivfibrillen innerhalb einer Zelle des Ganglion Nervi optici. (Schrägschnitt.) Vergrösserung wie bei Fig. 5. Bei $x$ ein annähernd quer abgeschnittener Protoplasmafortsatz. Die Fibrillen schlagen, nachdem sie in bündelförmiger Anordnung in die Zelle eingetreten sind, gewundene Wege ein, wobei sie vielfach mit einander verkleben. Hierdurch wird die gitterähnliche Structur hervorgerufen.

(Aus dem anatomischen Institut in Strassburg.)

\title{
Weitere Mittheilungen über den Bau der Hornschicht der menschlichen Epidermis und ihren sog. Fettgehalt.
}

\section{Von}

\section{Dr. Franz Weidenreich,} Assistent am anatomischen Institut.

Hierzu Tafel XXX u. XXXI und 1 Textfigur.

In meiner Arbeit ${ }_{n}$ Ueber Bau und Verhornung der menschlichen Oberhaut" (33) habe ich die Ansicht ausgesprochen, dass das Eleidin in den obereu Lagen der Hornschicht Umwandlungen erfahre, dic sich besonders in Aenderungen seines Aggregatzustandes zu erkennen geben würden; den so entstehenden festeren Körper habe ich als Pareleidin bezeichnet und ihn als das anatomische Substrat der färberischen Differenzen in den verschiedenen Schichten des Stratum corneum der Vola manus und Planta pedis angesprochen. Weiterhin hatte ich der Vermuthung Ausdrnck gegeben, dass die Befunde, welche anf die Anwesenheit von Fett in dieser Lage hinweisen, auf Reactionen jener Substanz zurtick- 
„uführen seien. Meine Untersuchungen hieruber waren jedoch beim Abschluss jener Arbeit noch nicht soweit gediehen, um zu einem bestimmteren Urtheil gelangen zu lassen; ihre Resultate seien im Folgenden mitgetheilt.

Zur Darstellung des Eleidins fand ich unter den vielfach angegebenen Methoden neben der von Frickenhaus (9) mit Recht gerühmten Wasserblaufärbung als die beste und am ehesten zum Ziele führende die von Buzzi (4) empfohlene Färbung mit Congoroth, von dessen $1 \%$ wässriger Lösung man 3-4 Tropfen in ein Uhrschälchen destillirten Wassers gibt. Die zu untersuchende Epidermis der Sohlenhaut oder der Haut der Handfläche kann unmittelbar der Leiche entnommen oder auch vorher einige (4-5) Stunden lang in 60-70\% Alkohol gehärtet werden; die Schnitte fertigt man am besten mit dem Rasirmesser aus freier Hand, jedoch erhält man auch bei Benützung des Gefriermikrotorns noch gute Resultate. Die Schnitte kommen sofort auf 3 bis 5 Minuten in die Farblösung, werden in Wasser gehörig abgewaschen und dann in Alkohol übergeführt; entstammen sie frischer, vorher nicht gehärteter Hant, so lässt man sie etwa bis zu einer Stunde im Alkohol, wodurch sie genugend fixirt werden, dann werden sie in Xylol gebracht und in Canadabalsam eingeschlossen; auf diese Weise hält sich die Farbe unverändert, wenigstens babe ich bis jetzt (8 Monate nach der Färbung) kein Abblassen beobachtet, während bei Aufbewalirung in Glycerin schon nach 1-2 Tagen eine diffuse Färbung des Schnittes eintrat.

Der Gehalt der Haut an Eleidin ist nun ein durchaus wechselnder; oft fand ich es überhaupt nur in Spuren, dann wieder sehr reichlich - selbstverständlich an denselben Hantstellen. Hierbei scheint etwas von Einfluss zn sein, wie lange nach dem Tode die betreffende Haut entnommen wurde, bei noch warmen Leichen habe ich im Allgemeinen das Eleidin stets in grösserer Menge gefunden als bei solchen, die erst mehrere Stunden nach dem Tode zur Untersuchung kamen; in den.letzteren Fällen gibt sich die bereits eingetretene saure Gewebsreaction sofort durch eine vorubberyehende Blaufärbung der Congorothlösung zu erkennen.

Was das Aussehen des Eleidins betrifft, so zeigt es sich in der Regel in Kugeln von wechselnder Grösse (Fig. 1, 2 und 3), oft finden sich mehrere kleinere wie Perlschnüre aneinander- 
gereiht (Fig. 3). Diese Kugeln haben fast stets einen tiefer gefärbten peripheren Ring und ein schwächer tingirtes Centrum (Fig. 1a); sehr häufig trifft man auch länglich ovale Formen, die dann, neben einander gelagert, lange Reihen bilden (Fig. 1b). Immer lässt sich jedoch beobachten, dass das Eleidin, ob es nun im einzelnen kugelig oder cval, gross oder klein ist, abgerundete Conturen aufweist, wodurch es sich scharf rom Keratohyalin unterscheidet, und ferner, dass die einzelnen Kugeln fast niemals uber die Grösse einer Zelle hinausgehen. Dass das Eleidin lange continuirliche, d. l. nicht von Eirschnürungen unterbrochene Streifen oder Bänder bildet, wie von einigen Autoren, so besonders von Buzzi, angegeben wird, konnte ich nicht beobachten. Derartige Bänder erwiesen sich stets als aus aneinandergereihten ovalen Gebilden zusammengesetzt, die in der Grösse mit dem Umfang einer Zelle der basalen Hornschicht übereinstimmen. (Fig. 1b.)

Was die Lage betrifft, so findet sich das Eleidin, wie man sich ohne Weiteres durch verschiedene Einstellung des Tubus ibberzeugen kann, auf beiden Sieiten des Schnittes. Es kann also seiner Form und Lage nach keinem Zweifel unterliegen, dass es von flüssiger Consistenz ist und infolgedessen bei dem Anschneiden der Zelle aus dieser heraustritt und so der Färbung zugänglich wird, wie das von dem Entdecker derSubstanz Ranvier (22) schon längst angegeben wurde. So erklären sich wohl die Kugelformen, nicht aber die ovalen Figuren; dass diese stets die Conturen der Zelle wiederholen und in Reihen nebeneinander liegen, obne jedoch ineinander zu fliessen, spricht dafür, dass hier das Eleidin in der angeschnittenen Zelle selbst gefärbt sein muss. Diese Annahme erhält eine weitere Stütze einmal dadurch, dass bei Haut, die einer schon längere Zeit gelegenen Leiche entstammt, das Eleidin fast nur in dieser Form aufzutreten pflegt, und dann ferner, dass man bei Haut, die mit Tannin fixirt wurde, auf dessen besondere Wirkung ich noch zurückzukommen haben werde, innerhalb des Stratum lucidum Zellen antrifft, die sich intensiv mit Congoroth färben, mit Ausnahme einer centralen runden oder ovalen Stelle, der Kernhöhle, und die genau den Form angenommen hat (vergl. Fig. 4 und Fig. 1 b). Aus der angeschnittenen Zelle tritt also das Eleidin in Tropfenform aus, ist jedoch auch, wenn es in derselben liegen bleibt, der Färbung zugänglich. Dass der letztere Fall bei nicht melır ganz frischer 
Haut der häufigere ist, lässt vermuthen, dass das Eleidin nit dem Erkalten iu etwas seinen flüssigen Aggregatzustand einbüsst und dann schwerer aus der Zelle ausläuft.

Hinsichtlich seines Vorkommens in der Hornschicht ist leicht festzustellen, dass es sich vor allem im basalsten Theile findet, entsprechend dem Stratum lucidum, wo es 2-3 Zellreihen ausmacht. Jedoch ist es keineswegs auf diese Zone beschränkt; wie Buzzi (4) zuerst richtig bemerkte und auch Dreysel und Oppler (6) bestätigt haben, begleitet es die Ausführgänge der Schweissdrusen fast durch das ganze Stratum corneum hindurch und setzt sich andererseits mit diesen auch etwas in das Stratum granulosum, bezw. germinat. hinein fort. Auch an diesen Orten zeigt es die Eigenthümlichkeit, kleine Tropfen zu bilden, die jedoch die des Stratum lucid. an Grösse nicht erreichen, sondern vorwiegend feine punktförmige Reihen auf den platten Zellen bilden, die im Kreise die Ausführgänge umgehen; diese Zellen erscheinen oft auch in toto lebhaft roth gefärbt und contrastiren dadurch ron denen des benachbarten Strat. corn.; nach dem oben Gesagten wäre auch hier das Eleidin in den Zellen selbst tingirt worden. Ausser an diesen beiden genannten Stellen ist es mir nun aber auch gelungen, innerhalb der mehr mittleren Schicht des Strat. corn. eine verhältnissmässig breite Eleidinlage aufzufinden, deren Oberfläche sich mit zahlreichen kleineren und grösseren Tröpfchen wie besäet erweist (Fig. 2 st). Diese Tröpfchen unterscheiden sich in ihrer Form von denen des Strat. lucid. dadurch, dass sie im Allgemeinen etwas kleiner sind und eine grosse Neigung besitzen, sich zu perlschnurartigen Kettchen aneinanderzulegen (Fig. 3), ohne dass jedoch auch hier eine solche Reihe die Länge einer Zelle uherschreitet. Daneben bestehen noch Verschiedenheiten in der Dichte ihrer Anordnung und ihrer Färbbarkeit. Der Raum, auf dem sich hier das Eleidin findet, umfasst etwa 10 Zellreihen; nur sind die Tropfen über dieses Gebiet ziemlich zerstreut und liegen nicht so reichlich und nahe beisammen wie im Strat. lucid., auch nehmen sie den Farbstoff weniger leicht an und färben sich picht so intensiv (Fig. 3), dagegen fällt die ganze Schicht schon bei schwacher Vergrösserung durch ihre stärkere Färbung auf, wodurch sie sich von den Nachbarlagen unterscheidet. Die Grenzen gegen diese sind wenn auch nicht scharf, so doch immerhin deutlich genug abgesetzt; 
sowohl nach aussen als nach innen bleiben die Tropfen im Wesentlichen auf eine bestimmte Zone beschränkt (Fig. 2 und 3). Nach innen folgt eine Lage von ca. 5-6 Zellreihen, die vereinzelte Tröpfchen aufweist (Fig. 2 und $3 s r$ ), nach aussen folgt die äusserste periphere Schicht (Fig. 2 und $3 s d$ ), in der sich keine Tropfen mehr finden. Diese Beschränkung auf eine ganz bestimmte Zone schliesst aus, dass es sich um eine Verschleppung des Eleidins aus dem Stratum Iucid. handeln könnte, weil dann doch auch die dazwischen gelegene Lage mit verschleppten Tröpfchen besprengt sein müsste; ferner spricht aber hauptsächlich gegen diesen Einwand, dass auch bei der Messerführung von der Peripherie nach der Cutis zu das Bild dasselbe bleibt. Es kann demnach keinem Zweifel unterliegen, dass an frischer oder kurz in Alkohol gehärteter und mit freier Hand geschnittener Haut sich ausser dem Strat. lucid. noch eine breite Zone innerhalb) der Horuschicht findet, aus deren Zellen beim Anschneiden der Inhalt heraustritt, und so der Färbung mit bestimmten Farbstoffen (Congoroth, Wasserblau) zugänglich wird. Dieser Zellinhalt charakterisirt sich dadurch, besonders aber auch durch seine Form (Tropfen) als ein flüssiger; seinem ganzen Verhalten nach stimmt er demuach mit dem Eleidin des Strat. lucid. überein.

Wir können also an dem strat. corn. vier Zonen unterscheiden (vergl. Fig. 2):

1) eine basale, das Stratum lucidum, mit reichlichem Eleidingehalt - Zone $\mathrm{A}(s l)$;

2) eine auf diese folgende Lage, ab und zu mit Spuren von Eleidin - Zone B (sr);

3) eine weiter peripher gelegene Schicht mit wieder reichlichem Eleidingehalt - Zone C $(s t)$;

4) eine oberfächliche Lage ohne nachweisbares Eleidin Zone $\mathrm{D}(s d)$.

Ich werde einstweilen diese Bezeichnung nach Zonen beibehalten, weil sie die Beschreibung vereinfacht; inwieweit das Gebiet derselben sich mit der bisher übtichen, von Unna (29) eingeführten Eintheilung deckt und ob sie überhaupt eine Grundlage für eine Eintheilung abgeben können, wird weiter unten zu erörtern sein.

Der oben beschriebene Befund ist nun aber keineswegs ein durchaus regelmässiger. Wäbrend sich bei einzelnen Individuen 
reichliche Mengen von Eleidin in der Zone $\mathrm{C}$ befinden, ist es bei anderen spärlicher vorhanden und oft nur in Spuren nachweisbar; fast stets bemerkt man dann aber in diesen Fällen eine mehr diffuse, stärkere Färbung der einzelnen Zellen jener Zone, die sich dadurch von den schwächer tingirten Nachbarschichten mehr oder weniger deutlich abhebt. Dieser wechselnde Eleidinwehalt der peripheren Zone steht aber etwas in Verhältniss zu der Menge des Eleidins in Strat. lucid.; tinden sich hier viele Tropfen, so sind sie auch in der oberen Lage in grösserer Zahl vorhanden; finden sich hier dagegen wenige oder keine, so fehlen sie auch dort. Hie und da kann man in der äussersten Schicht - Zone D intensiver gefärbte, unregelmässige kürzere Bälkchen oder Krümmel im Bereich einer Zelle nachweisen, die oft den Eindruck von Farbstoffniederscblägen machen, die aber, wie man sich durch entsprechende Einstellungr des Mikroskops iiberzeugen kann, innerhalb der Zellen liegen.

Um zu einer Deutung all dieser Befunde zu kommen, ist es nöthig, das Verhalten der Hornschicht am fixirten Material zu untersuchen. Je nach dem dazu verwandten Reagens wird das Bild ein verschiedenes sein. Härtete ich in Alkohol, so konnte ich entgegen den Angaben von Dreysel und Oppler (6) keine Eleidintropfen mehr nachweisen; im Gebiete des Strat. Iucid. fanden sich allerdings kleine Krümmel auf den Zellen liegend, die jedoch nichts für flüssiges Eleidin charakteristisches mehr zeigten. Nach diesen Autoren würde „gewöhnliche, nicht zu lang dauernde" Alkoholhärtung keine Consistenzveränderung des Eleidins bewirken; als Dauer der Härtung in absolutem Alkohol wird von ihnen 2-3 Tage angegeben. Meine Hautstitcke verblieben nur 24 Stunden in diesem Medium, allerdings dafür ziemlich lange (bis 8 Tage) in Celloidin, sodass möglicherweise die Differenz in den beiderseitigen Beobachtungen sich dadurch erklärt, für den Aufenthalt in Celloidin geben Dreysel und 0 p pler keine Zeit an. Nach Frickenha us (9) soll sogar noch nach einer vierteljährigen Alkoholhärtung (absoluter?) das Eleidin seinen flüssigen Charakter behalten. Wie den auch sei, ich halte diese Frage aus später anzuführenden Grüden nicht fur principiell bedeutungsvoll. Jedenfalls steht fest, dass nach lang Nauernder Alkoholeinwirkung, gleichgiltig ob man mit trockenem oder befeuchtetem Messer schneidet, keine Eleidintropfen 
mehr auftreten. So erhebt sich die Fragre, was aus dem Eleidin geworden ist; da giebt es nun zwei Möglichkeiten: entweder es ist in Lösung gegangen, oder es hat seinen Aggregatszustand derart verändert, dass es beim Anscheilen der Zelle nicht mehr herauslauft, d. h. dass es fest greworden ist. Diese letztere Ansicht vertritt Grosse (8), während Frickenhaus zu der ersteren Annahme neigt. Ich trete hier vollständiy auf Grosse's Seite; würde nämlich thatsächlich eine Lösung eintreten, so mïsste dies doch in der Structur der Zelle zum Ausdruck kommen, und da sie aus Membran, Fasernetz und dem dieses erfüllenden Eleidin besteht, so würde bei der Lösung des letzteren das Maschenwerk hervortreten müssen. Dies ist nun aber bei den Alkoholpräparaten nicht der Fall, vielmehr erweisen sich die Zellen diffus ron einer homogenen Masse erfüllt, wie ich es in meiner vorbergebenden Arbeit in den Fig. 15, 26 und 28 abgebildet habe, d. h. d a s Ele i d in hat, jedenfalls infolge der wasserentziehenden Wirkung des Alkohols, eine feste Consistenz angenommen; eine wesentliche färberische Differenz tritt in den einzelnen Schichten nicht mehr auf, die gesammte Hornschicht färbt sich fast gleichmässig.

Ganz anders gestaltet sich das Bild, wenn man zur Fixirung eine $1 \%$ ige Tanninsäurelösung benutzt (24 Stunden bei ca. $30^{\circ}$, Auswaschen in fliessendem Wasser, Celloidineinbettung). Fürbt man einen derartigen Schnitt mit Hämalaun und Congoroth (Fig. 5 ), so treten die Keratohyalingranula innerhalb des Strat. granul. deutlich hervor, während das Strat. corneum ein eigenthümliches Aussehen bietet. Unmittelbar auf die körnchenführende Schicht folgt nämlich eine ca. 10 Zellreihen hohe Lage (Fig. 5. $s l$ und sr), in der roth gefärbte Zellen auf bläulichem Grunde hervortreten. Diese Zellen sind bläschenförmig, zeigen eine deutliche Membran $(m)$, ein Faserwerk $(f)$ in ihrem Innern und eine centrale Kernhöhle (c), in der sich in den tiefsten Lagen noch ab und zu Kernreste $(n)$ erhalten haben (Fig. 5 und 6). Membran und Net\%werk nehmen mit Congoroth oder auch mit Pikrocarmin geenau dieselbe Farbe an wie das Eleidin und lassen sich auch mit Eosin sehr schön darstellen. Bei starker Vergrösserung machen diese Zellen den Eindruck, als wenn ihre Wände und die Fasern voneinander gezogen worden wären und nun eine schmierige, stärker lichtbrechende Masse sich zwischen diesen Fäden aus- 
spannen und an ihnen festkleben wïrde (Fig. 6). Bei kitrzer dauernder Tannineinwirkung tritt das Fasernetz weniger deutlich oder gar nicht hervor; man erhält dann Bilder, wie ich sie in Fig. 4 wiedergegeben habe, die sich von den mit der typischen Eleidindarstellung gewonnenen (Fig. 1) nicht unterscheiden. Die erst beschriebene Erscheinung ist jedoch nicht auf die unteren Zellreihen, die hier nicht nur das Strat. lucid., sondern auch die darüber gelegene '/one umfassen, beschränkt, sondern findet sich ebenso ausgesprochen an den seitlichen Schnitträndern in der ganzen Höhe des Strat. corm.; in der oberflächlichen Zone tritt dagegen das geschilderte Aussehen der Zellen weniger scharf hervor, Netzwerk und Membran werden hier nur an einzelnen Zellen deutlich, etwas häufiger die Kernhöhle, während au Stelle der schmierigen Masse nur kleine Krümmel nachweisbar sind. Der übrige Theil der Hornschicht zeigt mit Ausnahme der Seitenränder eine diffuse Blaufärbung, wie an stark gehärteten Alkoholpräparaten. Die Abgrenzung gegen dieses Gebiet ist sowohl von oben und unten als auch von den Seiten her keine scharfe. Ich glaube, dass für die Erklärung dieses Bildes die folgende Annahme die richtige ist: die Tanninlösung dringt von allen Seiten her in die Hornschicht ein und verursacht eine Quellung der Zellen bezw. ihres Inhaltes; diese nehmen so eine bläschenförmige Gestalt an, und das Eleidin spannt sich zwischen den Fäden des Zellinnern aus. Indem so durch die Quellung und Fixirung ein fester Gürtel gebildet wird, kann die Flüssigkeit nicht zu dem centralen Theil der Hornschicht gelangen und seine Zellen nicht zum Aufquellen bringen. Dabei scheint das Tannin die Eigenschaft zu haben, mehr auf den Zellinhalt als auf die verhornte Membran zu wirken, da die oberflächliche Schicht zum Unterschiede von den angeschnittenen Seiten nur wenig bläschenförmige Zellen aufweist; die Krümmel deuten hier rielmehr darauf hin, dass das Eleidin hier völlig eingetrocknet ist und damit auch seine Quellungsfähigkeit verloren hat. Dafür sprechen noch andere, später zu erwähnende Beobachtungen. Dass die \%wischen dem Maschenwerk des Zellinnern ausgespannte schmierige Masse wirklich das durch das Reagens allerdings in seiner Consistenz veränderte Eleidin ist, geht einmal daraus hervor, dass das ganze Strat. lucid. diese Eigenthïmlichkeit zeigt (Fig. $5 s l$ ) und dass 
bei kurz dauernder Fixirung sich einzelne Zellen noch mit unverändertem Eleidin gefüllt erweisen (Fig. $4 e$ ).

Fixirt man Solılenhaut nach den Angaben Unna's (32) in einer Lösung von 1,0 gr 'Tanninsänre und 1,0 Pikrinsäure in 100 ecm eincr $1 \%$ igen Salpetersäurclösung bei einer Temperatur von ca. $30^{\circ}$, bettet die so behandelte Haut dann in Celloidin ein und schneidet genau senkrecht zu den Riffen der Oberfläche, so erhält man bei Färbung mit Congoroth oder Pikrokarmin folgendes Bild (Fig. 7): Die Hornschicht lässt sich deutlich in vier Zonen abgrenzen; die unterste Zone $A$, dem Strat. lucid. entsprechend $(s l)$, ist intensiv gelb gefärbt, die Zellen selbst sind stark abgeplattet. Darauf folgt eine breite stark roth tingrirte Zone B $\left(s r^{\circ}\right)$; hier sind die Zellen bläschenförmig und lassen eine dentliche Membran und Fasernetz, sowie eine leere Kernhöhle erkennen (in Figur $\tau$ wegen der schwachen Vergrösserung nicht wiedergegeben); älnnlich wie bei den Tanninpräparaten spannt sich zwischen den Fasern eine stark roth gefärbte, schmierige Masse aus, das gequollene Eleidin. Auf diese Schicht folgt eine Lage (C) gelb gefärbter platter Zellen (st), an denen keine Structur zu erkennen ist und darauf wieder eine Zone (D) bläschenförmiger Zellen (sd) mit Menbran, Fasernetz und leerer Kernhöhle und zwischen dem Faserwerk eine mehr krümmlige Masse. Der Uebergang von einer Zone in die andere ist zwischen $A$ und $B$ sehr scharf, weniger \%wischen $B$ und $C$ and $C$ und D. Neben dieser verschiedenen Schichtung fällt nun sofort anf, dass im Gebiet der die oberflächlichen Riffe begrenzenden Furche, also in dem von Blaschko (2) beschriebenen Faltentheil die Zone C der platten Zellen sich gegen das Strat. Malpighi hin einsenkt und das Strat. lucid. (Zone A) erreicht (pi). Die Hornschicht erscheint also hier, wie übrigens schon $0 \mathrm{ehl}(19)$ beobachtet und abgebildet hat, deutlich in den intrapapillären Theil des Strat. Malpighi eingefaltet, nur die oberflächliche Schicht ist dabei weniger betheiligt und weist nur über der Falte eine Einbuchtung auf (in Fig. 7 nicht ausgeprägt). Behandelt man einen derartig fixirten Schnitt nach den weiteren Unna'schen Angaben mit einer Osmium-Alaunlösung, so färbt sich Zone $\mathrm{A}$ und $\mathrm{C}$ intensiv olivengrün (Fig. $8 s l$ und $s t$ ), wodurch die platten Zellen von den heller osmirten bläschenförmigen der anderen Lagen (sr) scharf contrastiren; in Faltentheil $(p i)$ erkennt man so besonders deut- 
lich, dass hier die Zellen statt mit ihrem grössten Durchmesser horizontal orientirt zu sein, sich der Faltenrichtung entsprechend vertical gestellt haben. Dasselbe Verhalten hat nun bereits Zander (35) beobachtet; er giebt davon in Fig. 4 (Taf. V) ein anschauliches Bild. Dieses Z ander'sche Präparat entstammt Haut, die in Chromsäure gehärtet und mit Methyleosin gefärbt war. Wir haben dort eine stark roth gefärbte Zone A (Strat. lucid.) mit platten, eine Zone B mit bläschenförmigen Zellen, eine Zone $\mathrm{C}$ mit wieder mehr abgeplatteten, die sich im Gebiet der Falte zum Strat. Malpighi einsenken und endlich eine periphere Zone D mit bläschenförmigen Zellen, die bei der Einfaltung weniger betheiligt ist. Zander hat dieses Bild so erklärt, dass die stark roth gefärbten und abgeplatteten Zellen total verhornt wären (Typus B), während die bläschenförmigen Zellen den für Vola manus und Planta pedis charakteristischen Bau zeigen würden (Typus A). Aehnliche Bilder ergiebt nun auch die Zenkersche Flisssigkeit.

Bei der Deutung dieses Phänomens entsteht als nächste Frage: Handelt es sich hierbei um ein Kunstprodukt, d. h. wird die eigenthümliche Anordnung und Verschiedenheit der Zellen durch das Fixationsverfabren bedingt, oder finden sich diese Unterschiede auch an frischer Haut? Ich habe bereits anseinandergesetzt, dass sich thatsächlich an frischer Haut Differenzen insoweit nachweisen lassen, als wir je nach dem Auftreten des Eleidins vier Zonen unterscheiden können. Aber auch die Falte lässt sich ohne Weiteres nachweisen, nur erscheint die oberflächliche Schicht stärker eingebuchtet, dies entspricht eben der Furche zwischen den Riffen der Oberfläche. Diese Verhältnisse hat O ehl (19) nach Beobachtungen an frischer Haut in Taf. I Fig. 3 and 5 schon vor bald 50 Jahren abgebildet. Auch an Haut, die kurze Zeit in $60-70 \%$ Alkohol gelegen hat, lassen sich diese Verhältnisse noch gut erkennen, sehr laug dauernde Alkoholeinwirkung verwiseht dagegen das Bild, eine Beobachtung, die auch Unna (29) bestätigt. Dagegen kann weder an frischer noch an kurz in Alkohol gehärteter Haut in der Zellstructur der verschiedenen Schichten ein Unterschied wahrgenommen werden; niemals treten hier bläschenförmige Zellen mit Membran und Fasernetz hervor. Ibr Auftreten ist also auf eine Quellung zurick- 
zuführen, wohl mit theilweiser Lösung des Zellinhaltes, d. h. des Eleidins; diese Quellung wird thatsächlich durch die zur Fixirung benutzte Säurelösung bedingt, in meinen Fällen durch die Salpetersäure, Essigsäure und die M üll e r'sche Flüssigkeit, bei $\mathrm{Z}$ and er durch dic Chromsäure.

Wie kommt es nun, dass diese Quellung sich nicht auf die ganze Hornschicht erstreckt, sondern nur auf bestimmte Lagen derselben? Da wir im Grossen und Ganzen auch an frischer Haut wesentliche Unterschiede finden, mïssen diesen Ursachen zu Grunde liegen, die in einem besonderen Zustande des Stratum corneum zu suchen sind; diese können aber nichts anders als Spannungsunterschiede sein, welche ich an dem folgenden Schema erläutern will.

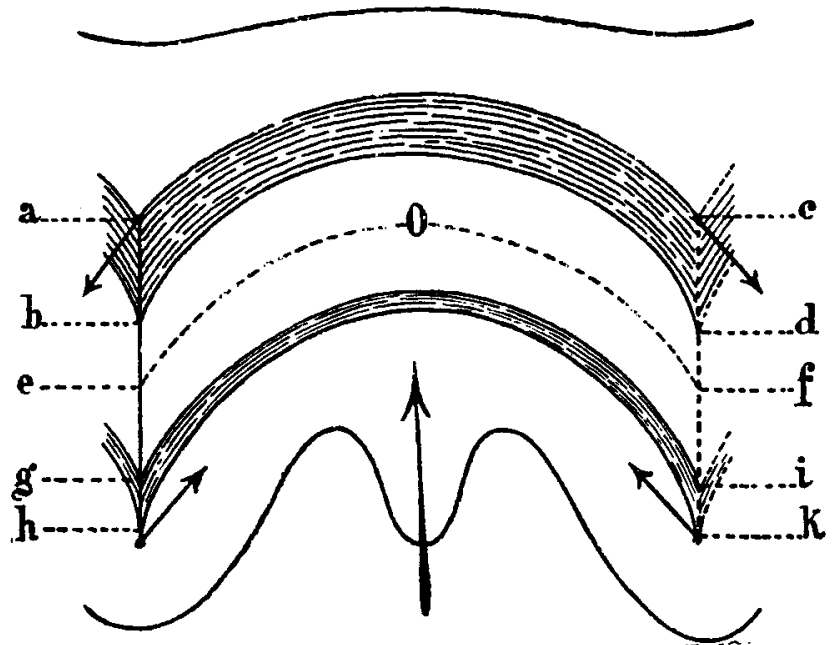

Schema zur Erläuterung der Spannungsverhältnisse im Stratum corneum von Vola manus und Planta pedis. $a h$ und $c k=$ Zugrichtung im Blaschko'schen Faltentheil nach innen. Grosser Pfeil in der Mitte = Gegendruckrichtung nach aussen. Pfeile bei $h$ und $k=$ Richtung der die Zone $g h i k$ (Zone A) zusammenpressenden Kraft. Pfeile bei $a$ und $c=$ Richtung der die Zone $a b c d$ (Zone C) spannenden Kraft. $O=$ Interferenzzone (Zone B); $e f=$ deren Mittellinie.

Die Hornschicht ist im Gebiete der Blaschko'schen Falten gegen die Cutis zu stark eingezogen, während die zwischen zwei Falten gelegene Partie, die der Drüsenleiste entspricht, 
nach der Oberfläche au vorgewölbt ist; dadurch wird in diesen Theilen eine dauernde Spannung erzeugt, welche sich in folgender Weise äussert: Nelımen wir in der Richtung der Faltenlinie $a h$ und $c k$ einen gegen die Cutis wirkenden Zug an und dementsprechend im Gebiete des Drüsenleistentheils von der Cutis her einen durch den mittleren Pfeil angedeuteten Druck nach aussen, so entstehen in dem Gebiete $a c h k$ Spannungsunterschiede und zwar werden in der Zone $g h i k$ die Zellen gegen die Mitte zu in der Richtung der Pfeile bei $h$ und $k$ zusanmengepresst, während in der Zone $a b c d$ umgekehrt die Zellen von der Mitte nach den Seiten auseinander gezogen werden. Darlurch entsteht, da ja die Linien $a h$ und $c k$ sich einander nähern können, nach dem Gesetze eines an seinen Enden frei beweglichen Stabes eine Interferenzzone $b d g i$ mit der Mittellinie $e f$, in welcher die Spannung gleich Null ist. Einer grossen Spannung unterliegen demnach einmal die Zellen der Zone $g h i k$ (Zone A, Stratum lucidum) und der Zone $a b c d$ (Zone $\mathrm{C}$ ), ebenso die Zellen des Faltentheils $b g$ und $d i$, keiner dagegen die der zwischen diesen Linien gelegenen Zone $b g d i$ und der an der Einfaltung nicht mehr betheiligten äussersten peripheren Schicht (Zone D). Die Zonen $A$ und $C$ haben demnach ein festes Gefüge, die einzelnen Zellen unterliegen einer starken Span$\mathrm{n} \mathfrak{u} \mathrm{g}$ in horizontaler Richtung, während die $\mathrm{Z}$ on en $\mathrm{B}$ und $\mathrm{D}$ locker geschichtet sind, auf ihre Zellen alsokein bes onderer $\mathrm{Zug}$ einwirkt.

In diesen Spannungsverhältnissen der einzelnen Schichten finden wir nun eine unzweideutige Erklärung für die Bilder, wie sie die verschiedenen Fixirungsmethoden liefern. Bei all den Mitteln, die Quellung verursachende Reagentien enthalten, wie die Unna'sche Lösung, die Chromsäure, die Zenker'sche Flüssigkeit etc., äussert sich diese Quellung zunächst in den lockeren Schichten, weil hier die Flüssigkeit am leichtesten eindringen und sich ausbreiten kann, die Zellen nehmen dadurch Bläschenform an und werden so fixirt; während dort, wo ein festes Gefuge besteht, die Flüssigkeit schwerer und später eindringt, so dass diese Zellen durch die bereits gequollenen und fixirten der Nachbarlagen an der Ausdehnung verhindert werden, ja vielleicht sogar durch jene eine Zusammenpressung erfahren. So erklärt sich, dass die Zonen $A$ und $C$, sowie die Blaschko- 
sche Falte stark abgeplattete Zellen aufweisen, die infolge dessen auch färberische Differenzen zeigen werden, indem sie sich mit bestimmten Farbstoffen (Carmin, Congorot, Methyleosin) intensiver tingiren lassen. Verwendet man zur Fixirung dagegen Reagentien, die keine Aufquellung bedingen, wie den Alkohol, so kommen die Unterschiede der Schichten weniger scharf zum Ausdruck; man findet hierbei keine bläschenförmigen Zellen, sondern durchweg mehr oder weniger abgeplattete, deren Inhalt in eine feste homogene Masse umgewandelt worden ist; doch bleibt die $\mathrm{Bl}$ a s c h k o'sche. Falte deutlich ausgeprägt. Bei ganz frischer Haut ist sie dagegen oft etwas weniger bemerkbar, hierbei trifft man dann eine mehr wellenförmige Anordnung derart, dass die Wellenthäler stets in Gebiet der Falte gelegen sind. Diese geringe Verschiedenheit ist darauf zurückzufiihren, dass bei frischer Haut infolge der ihr innewohnenden bedeutenden Elasticität die natïrliche Spannung durch das Ausschneiden des dünnen Rasirmesserschnittes etwas verloren geht. Ist nun der Inhalt der Hornzelle flüssig, wie bei frischer oder kurz in Alkohol gehärteter Haut, so wird derselbe beim Anschneiden das Bestreben haben, a us der Zelle a uszutreten, und zwar in den Schichten, wo die Zellen einer besondere $\mathrm{n} S \mathrm{~S}$ an $\mathrm{n}$ ung ihrer W and $\mathbf{n}$ g unterworfen sind. Dies ist nun, wie auseinandergesetzt, der Fall in den Zonen $\mathbf{A}$ und $\mathbf{C}$, in den Lagen also, wo sich nach meinen eingangs angeführten Beobachtungen Eleidintropfen in reichlicher Menge nachweisen liessen; dagegen wird in der Interferenzzone B, ebenso wie in der oberflächlichen Lage D, da hier die pressende Kraft fehlt, nichts aus der Zelle ausfliessen. Es ist nun selbstverständlich, dass diese Zonen verschiedener Spannung sich nicht scharf von einander abgrenzen, mit Ausnahme des Uebergangs von Zone $A$ in $B$; hier tritt eine schärfere Trennung hervor, weil an dieser Stelle das Eleidin im Allgemeinen in eine festere Form tibergeht. Aber auch dieser neuentstandene Körper kann noch hier und da ausgepresst werden, das ist dann der Fall, wenn die Spannungsverhältnisse gut ausgeprägt sind und sein Aggregatszustand nicht allzufest ist. Ist dagegen die Spannung eine geringere und das Eleidin wenig liquid, so gelingt es nicht, es noch in den höheren Lagen nachzuweisen. 
Die geschilderten Spannungsunterschiede können nun noch durch eine rein physikalische Eigenschaft der Hornschicht sichtbar gemacht werden, auf die von Ebner (7) schon längst aufmerksam gemacht hat, die ich aber merkwürdiger Weise in keiner Beschreibung der Epidermis erwähnt finde, das ist die Anisotropie des Stratum corneam. Untersucht man Hornschicht, die nach $U \mathrm{n} n$ a fixirt ist, im polarisirten Licht, so erkennt man leicht, dass die Zonen $\mathrm{A}$ und $\mathrm{C}$ ausserordentlich stark doppellichtbrechend sind, bedeutend weniger dagegen die úbrigen Zonen; lange nicht so in die Augen springend ist der Unterschied bei frischer Haut, weil die Elasticität hier die Spannung ausgleicht; immerhin aber tritt sie deutlich genug in den Zonen $\mathrm{A}$ und $\mathrm{C}$ und in den gegen die $\mathrm{Blaschko}$ 'sche Falte geneigten Zellreihen hervor, besser noch wenn man die Hant etwas eintrockuen lässt oder ein kurz in Alkohol gelegtes Stück verwendet. Dagegen erweist sich nach lang dauernder Alkoholhärtung das Strat. corneum fast gleichmässig anisotrop; behandelt man aber einen derartigen Schnitt mit Essigsäure, so quellen die Zellen auf und in demselben Masse nimmt in den gequollenen Partien die Doppellichtbrechung ab, eine Beobachtung, die zu dem Schlusse berechtigt, dass nicht gequollene und gespannte Zellen je nach dem Grade der Spannung anisotrop sind. Ich habe mich bemüht, festzustellen, an welchen Zellbestandtheil die Doppellichtbrechung gebunden ist, an die Hornsubstanz oder an das Eleidin. Die erstere kann jedenfalls anisotrop sein, wovon man sich ohne Weiteres überzengt, wenn man ein dünnes Strickchen Nagel zwischen die Nicols bringt; die Ränder zeigen sich dann isotrop, die Mitte mehr anisotrop. Dagegen fällt für das Eleidin der Nachweis schwerer; an den Tropfen lässt sich mit Bestimmtheit nichts feststellen, dagegen erweist sich der gesammte Inhalt der Stratum lucidum-Zellen sehr stark doppellichtbrechend, ein Umstand, der mir doch dafür ıu sprechen scheint, dass auch das Eleidin, wenigstens wenn es sich in einem gepressten Zustande befindet, anisotrop ist.

Die Spannungsunterschiede der Hornschicht erklären nun aber auch ein Phänomen, das neuerdings von Merk (16) beobachtet wurde, von ihm aber, wie mir scheint, nicht in zutreffender Weise gedentet wird. Merk fand nämlich, dass bei 
Injectionen in die Fingerbeere eines frisch amputirten Fingers, gleichviel mit welcher Flüssigkeit, eine ${ }_{n}$ Verbreiterung ${ }^{*}$ der über dem Strat. lucid. gelegenen Zone eintrat, deren Zellen aufquollen und Bläschenform annahmen. Ueber den Drüsenleisten war die Verbreiterung stärker, schwach an den anderen Stellen, sodass an den ${ }^{\text {von }}$ der Injection mehr entfernten Partien nur die erstere Art der Verbreiterung (d. h. eine Beschränkung auf die Drüsenleiste. D. Ref.) bei schwachen oder mässigen Vergrösserungen entdeckt werden konnte ${ }^{4}$. Die Merk'schen Figuren 1 a und 7 lassen ohne Weiteres erkennen, dass seine "Verbreiterungszone ${ }^{\text {" }}$ identisch ist mit meiner Zone B (vgl. Schema und bes. Fig. $9 \mathrm{sr}$ ), die, wie oben auseinandergesetrt, locker gefügt ist. Es ist klar, dass infolgedessen die Injectionsflüssigkeit sich in dieser Lage besonders leicht ausbreiten wird, nicht dagegen dort, wo eine starke Spannung herrscht wie in den Zonen A and B. Bei starkem Druck, d. h. also in der Nähe der Einspritzungsstelle wird dieser Widerstand tiberwunden und zwar zunächst in der Lage, in der er am schwächsten ist, also im unteren Faltentheil; die Flüssigkeit breitet sich dann auch hier aus. Bei geringerem Druck, d.h. bei grösserer Entfernung, bleibt sie dagegen auf die lockere Schicht beschränkt; sie wird hier diese Lockerung noch erhöhen und dem fixirenden Reagens das Eindringen in die Zone erleichtern; ist dieses säurehaltig, so bringt es sofort die Zellen zum quellen, thatsächlich rühmt nun auch M erk die Wirkung der Zenker'schen Flüssigkeit, die das Phänomen besonders zeige.

Ganz abweichend hiervon beurtheilt nun $M$ e $r k$ die mitgetheilte Erscheinung. Nach ihm handelt es sich dabei um eine Lebensthätigkeit der Hornzellen, die im Stande wären, ïberschïssige Flüssigkeitsmengen aus dem darunter liegenden Gewebe aufunnehmen und an die Oberfläche abrugeben; es käme ihnen so eine bedeutende „regulatorische Thätigkeit" zu. 'Trotzdem ich bereits in meiner ersten Abhandlnng gegen diese Annahme, wie ich glaube, schwerwiegende Bedenken geltend gemacht habe, hält $\mathrm{Merk}$ in einer eben erst erschienenen Arbeit (17) seine Behauptung aufrecht; er betont zwar ausdrücklich, dass ich seine Ausführungen nicht direct als gegen meine Person gerichtet ansehen möge, weil mein Standpunkt der allgemein bisher 
vertretene wäre; ich glaube aber dennoch das Recht in Anspruch nehmen zu dürfen, seiner Auffassung entgegenzutreten. Zunächst musste man sich eigentlich darüber verständigen, was beiderseits unter einer Lebensthätigkeit der Hornzelle verstanden wird. M e rk erläutert dies nicht näher, aber aus seinen Ausführungen geht hervor, dass er annimmt, die Hornzelle vermöge in dem vorliegenden Falle durch eine besondere Function ibres Protoplasmas Flüssigkeit activ aufzusaugen, in sich aufzuspeichern und nach Bedarf wieder abzugeben; das ist doch wohl das Wesen einer regulatorischen Thätigkeit. Daneben führt er noch an, dass die Hornzelle bei pathologischen Processen Veränderungen eingehe, über deren Natur aber einstweilen keine näheren Angaben gemacht werden; er erwähnt hier nur besonders die friihzeitige Abstossung der Zellen nach Aetzung und Verbrennung und meint, wenn die Zelle doch schon todt wäre, könnte sie nicht gut auf diese Schädlichkeiten reagiren und so noch "todter" werden. Derartige Einwände haben doch recht wenig Beweiskraft. Liegt es dann nicht viel näher, für diese Reactionen Aenderungen in den rein physikalisehen Eigenschaften der Hornzelle anzunehmen? Von diesen käme besonders in Betracht die Elasticität, die Quellungsfähigkeit und die Neigung des in den tiefen Schichten z. Th. flussigen Zellinhalts mit dem Vorrücken der Zelle nach der Oberfliche fest zu werden und einzutrocknen; eine vorzeitige Abstossung der Hornzellen kann ebensogut auf einer starken Lockerung des Zellverbandes durch rein mechanische Momente basiren. Das Merk'sche Phänomen ist leicht, wie bereits gezeigt, auf physikalische Eigenthümlichkeiten der Zellen zurück»uführen. Dass also die Hornzelle durch chemische und thermische Reize verändert werden kann, beweist gar nichts für eine Lebensthätigkeit derselben. Wenn ich z. B. ein Stück altes Horn vom Pferdehuf in Kalilauge koche, so bildet sich zunächst eine schleimige Masse; niemand wird aber deswegen sagen, das todte Stick Horn ist nun noch "todter" geworden; oder aber wenn ich ein Blatt von einem Baume abschneide und lege es an die Sonne, so vertrocknet es schliesslich zu einer dürren, brüchigen Masse; die Zellen waren aber schon bedeutend früher abgestorben, bevor dieses Endresultat eintrat, und auch in diesem ntodten" Zustande kann es, bezw. seine Zellen, noch "todter" gemacht werden, nämlich dann, wenn man es verbrennt. $\mathrm{Zu}$ - 
nächst wäre demnach zu prüfen, sind die von Merk angeführten Punkte nicht auf rein physikalische Eigenschaften der Hornzellen zurückzuführen, die, wenn auch abgestorben, vermöge ihres Reichthums an solchen auf äussere Einwirkungen sehr leicht reagiren können.

Als Haupteinwand gegen eine active Lebensthätigkeit hatte ich mit geltend gemacht, dass die Hornzellen des Kerns entbehren und dass es unseren physiologisehen Anschanungen widerstreite, einem kernlosen Gebilde wirkliches Leben zuzusprechen. Dagegen hebt nun Merk besonders hervor, dass er Kerne in den Hornzellen gefunden haje, die sich leicht färben liessen und dass in den Zellen seines Zehennagels solche gleichfalls nachweisbar waren. Ich weiss sehr wohl, dass man in den basalen Hornlagen $a b$ und zu gut färbbare Kerne trifft und dass dieser Befund für die Hornzellen des Nagels der normale ist; allein die Thatsache bleibt bestehen, dass in den höheren Lagen der Hornschicht Kerne normaler Weise niemals gefunden werden; aber auch die in den tiefsten Lagen des Stratum corn. nachweisbaren Kerne lassen alle eine deutliche Schrumpfung erkennen und sind im Begriffe zu zerfallen (Fig. $5 n$ ). Ganz aber fehlen sie in den Zelllamellen der nach dem von Zander (35) so benannten Typus $B$ gebauten Haut, d. h. an allen Hautstellen mit Ausnahme der Vola manus und Planta pedis. Merk ignorirt in seiner Erwiderung den von mir bereits früher gemachten Einwand völlig, und doch kommen für pathologische Processe praktisch wohl hauptsächlich diese Partien in Betracht, jedenfalls aber dann, wenn es sich um eine wichtige physiologische Function der Hornschicht handeln soll, wie die ${ }_{n}$ regulatorische Thätigkeit ${ }^{4}$ eine wäre. Ich muss also meine Frage wiederholen: Ist diesen Lamellen auch eine active Lebensthätigkeit zuzusprechen? Gelingt es hier überhaupt - darauf basirt ja in ihrem Kernpunkt die Merk'sche Theorie - Flüssigkeit in die Zellen selbst, nicht in die Interlamellarräume, mittels subepidermaler Injection hineinzuspritzen? Solange also keine stichhaltigeren Beweise für ein Leben der Hornzelle beigebracht werden können, wird sie wohl einstweilen noch ibre Auferstehung nicht feiern; es bleibt vielmebr dabei, dass die Epidermiszellen einer fortschreitenden Degeneration unterliegen, die schliesslich zum Absterben der Zelle flubrt ${ }^{1}$ ).

1) Es sei mir hier gestattet, obwohl es nicht direct im Rahmen 
Der auffallende färberische Unterschied zwischen den einzelnen Schichten des Strat. corn. hat nun Unna (29, 30) schon früher veranlasst, eine Theorie hierbiber aufzustellen; darnach würde es sich um Consistenzverschiedenheiten der Zelllagen handeln, was also im Princip mit meiner Aufstellung iubereinstimmen würde. Unna's Untersuchungen beschränkten sich in der Hauptsache auf Fixirung der Hant in Osmium oder Alkohol mit nachfolgender Pikrokarminfärbung. Auf Grund so gewonnener Bilder nimmt er an, dass das Strat. lucid. sehr fest gefügt sei, eine darunter gelegene "superbasale" Schicht fest, eine folgende "mittlere" locker und die ${ }_{n}$ oberffächliche ${ }^{\mu}$ wieder fest. Für die Aufstellung einer eigenen superbasalen Schicbt, die Unna als stark rothen, scbmalen Streifen nur bei Pikrokarminfärbung fand, ist kein zwingender Grund vorbanden; ausserdem aber geht aus meinen Osmiumprä-

des Themas liegt, kurz auch auf die Einwande Merk's (17) einzugehen, die sich auf den Bau der Hornzelle beziehen. Zunächst habe ich nirgends behauptet, dass sich zwischen den Hornzellen isolirte Fibrillen fändeu, die entfernter gelegene Zellen mit einander verbinden würden. Ich leugne doch sogar schon eine Verbindung benachbarter Hornzellen durch Fasern. Was nun die Frage nach den Poren der Zellen angeht, so hat Merk ein neues Argument dafür nicht vorgebracht, noch meine Einwände widerlegt. Oberfächenbildern kommt hier m. E. wenig Beweiskratt zu, da hierbei sich niemals mit Bestimmtheit sagen lässt, ob die Vertiefungen, wie sie sich in den Merk'schen Figuren A, B etc. finden, wirkliche Löcher oder nur Grübchen darstellen; die reproducirten Photographien lassen die letztere Annahme sogar für richtiger erscheinen, die Zellen bieten nicht den Anblick einer siebartig durchlöcherten Hülle, sondern eher ciner mit Zacken und Kämmen besetzten Oberfläche, die durch kleine Gruben von einander getrennt sind. Die Frage kann eben nur sicher entschieden werden mittelst eines Durchschnittes durch die Zelle; hierbei zeigt sich nun auf das Schlagendste, dass die Oberfläche mit Zähnchen besetzt ist, aber Durchbrechungen der Membran, wie sie doch beim Vorhandensein von Poren unfehlbar auftreten müssten, lassen sich nirgends, auch in den düunsten Schnitten von $2,5 \mu$ nicht, constatiren. (Ich verweise hier nur auf die Fig. 15, 16, 17, 18, 20 u. ff. meiner ersten Abbandlung.) Wenn es also gelingen sollte, auf einem Zelldurchscbnitt Lücken in der Membran aufzufinden, die in das Zellinnere hinein führen, daun erst wäre das Vorhandensein von Poren unwiderleglich bewiesen. 
paraten hervor, dass seine oberflächliche Schicht mit den von mir als Zone $\mathrm{C}$ und $\mathrm{D}$ bezeichneten nngefähr identisch ist. Beriticksichtigt man dies, so deckt sich die Unna'sche Angabe tiber die Consistenzrerschiedenheiten mit der meinigen; es würde also die basale feste Schicht der Zone A, die mittlere lockere der Zone $\mathrm{B}$, die oberflächliche der Zone $\mathrm{C}$ und meine lockere Zone D wohl dem peripheren Theile von Un $n$ a's obertlächlicher Schicht entsprechen. Allein in der Erklärung der Consistenzunterschiede weiche ich von $\mathrm{Unn}$ a ab; er hat ziemlich complicirte Theorien über die Aeusserung der Oberflächenspaunung auf die einzelnen Hornzellen aufgestellt. Wieweit diese in Betracht kommen, will ich dahin gestellt sein lassen; ich glaube, dass die von mir gegebene Deutung wesentlich einfacher ist, sie deckt sich zudem völlig mit den Befunden bei den verschiedensten Fixirungen und von frischer Haut.

Fasse ich nunmehr die Ergebnisse zusammen, so lässt sich ther den $\mathrm{Ba} \mathfrak{a}$ der Hornschicht folgendes sagen: Das Stratum corneum wird von Zellen gebildet, an denen sich eine verhornte Membran und im Innern ein Fasernetz unterscheiden lässt, das jedenfalls aus einer sehr resistenten Substanz, sicher aus irgendwie ve rä $n d$ e $\mathrm{t}$ e $\mathrm{m}$, jedoch nicht $\mathrm{v}$ erhorntem Protoplasma, hesteht; der übrige Zellraum wird von einer homogenen Masse eingenommen, die jenes Net\%werk a usfüllt. Diese Masse entsteht durch Verflüssigung der im Strat. gran. gebildeten Keratohyalingranula und heisst dann Eleidin; in den basalen $2-3 \mathrm{Zell}$ reihen der Hornsebicht behäl dieses Eleidin seinen flassigen Charakter bei, nimmt aber dann eine feste, zähe, colloidartige Consistenz an und trocknet in den 0 berflächenzellen zu einer krümmeligen Masse ein, wähend Membran und Faserwerk morphologisch keinerlei Veränderung mehr erfahren. Ich hatte in meiner ersten Abhandlung einzelne Beobachtungen aufgefuhrt, die für eine Betheiligung des Schweisses bei der Eleidinmetamorphose sprechen, konnte jedoch weitere Beweise für diese Annahme nicht finden; nahe liegt es jedenfalls die Wirkung der Luft fur die Austrocknug des Eleidins anzunehmen. Für die festere Form dieser Substanz babe 
ich den Namen Pareleidin vorgeschlagen, weil mit dem Uebergang des Eleidins in diesen Zastand auch chemische Umwandlungen einherzugehen scheinen, wofür die später zu erwähnende Osmiumreaction spricht. Demnach könnte man im Allgemeinen den homogenen Inhalt der Hornzelle jenseits des Strat. lucid. als Pareleidin bezeichnen. Neben dieser Structur der Zelle selbst bietet auch die Hornschicht als solche in ihrem Gefüge wesentliche Verschiedenheiten dar. Die basale Schicht - Zone A, Strat. lucid. - besteht aus stark abgeplatteten, festgefigten und gespannten Zellen, auf diese folgt eine lockere Schicht - die Interferenzzone B - mit weniger abgeplatteten Zellen, dann wieder eine feste Zelllage im Zustande der Spannung - Zone C - und endlich eine oberflächliche lockere Schicht mit den sich abstossenden Zellen - Zone D. Im Gebiet der Blaschko'schen Falte erstreckt sich die gespannte Zone $\mathrm{C}$ bis zum Strat. lucid. herab und unterbricht hier so die lockere Schicht B (vergl. Fig. 7, 8 und 9).

Ich habe bereits darauf hingewiesen, dass dieses eben geschilderte Bild individuellen Schwankungen unterworfen ist, die in einem mehr oder weniger bedeutenden Ausgleich der Spannungsdifferenzen oder in der früher oder später eintretenden Aenderung des Aggregatzustandes des Eleidins, bezw. des Pareleidins bestehen. Es wäre jedenfalls zur Erkenntniss pathologischer Vorgänge nicht ohne Werth, zu untersuchen, in wie weit Alter und Ernährungszustände diesen wechselnden Befunden zu Grunde liegen und in wie weit sie bei Krankheiten, welche die Hornschicht betreffen, eine Rolle spielen. Ein besonderes morphologisches Interesse bietet die angezogene Frage jedenfalls nicht. Vielleicht empfiehlt es sich vom praktischen Gesichtspunkte aus die einzelnen Schichten des Stratum corneum mit Namen zu belegen, die zugleich auch einen Hinweis auf ihren Bau enthalten; ich schlage also für den Fall, dass sich ein besonderes Bedürfniss nach einer dementsprechenden ausführlichen Nomenclatur herausstellen sollte, folgende Bezeichnungen vor (vergl. dazn Fig. 9): 
Stratum corneum.

Pars arcuata $\left.(p a)^{1}\right)$

Pars implicita (pi)

(Drüsenleistentheil)

(Gebiet der Blaschko'schen Falte)

A. Stratum lucidum (Oehl) (sive Stratum tensum profundum - Stratum basale Unnae) ( $s l)$;

B. Stratum relaxatum (s.

Stratum medium Unnae)

fehlt

$(s r)$;

C. Stratum tensum (bezw. tens. superfic.) (st)

D. Stratum disjunctum (Ranvier) (sd)

(C und D - Stratum superficiale Unnae).

Die Pars implicita unterscheidet sich also von der Pars arcuata dadurch, dass hier das Stratum relaxatum fehlt, an seiner Stelle reicht das Stratum tensum bis zum Stratum lucidum herab. In der Mitte der Pars arcuata verläuft durch alle Schichten hindurch der Ausführgang der Schweissdrüsen (ds).

Es erscheint mir nothwendig, auch hier wieder besonders hervorzulieben, dass in der eben geschilderten Weise $\mathrm{nur}$ die Hornschicht von Vola manus und Planta pedis gebaut ist, während das Stratum corneum der übrigen Hautstellen aus wenigen ubereinander geschichteten Lamellen besteht, die ab und $\%$ durch Spalträume von einander getrennt sind, welche man zweckentsprechend als Interlamellarräume bezeichnen könnte. Die Lamellen selbst wieder bestehen ans stark abgeplatteten, untereinander verklebten Hornzellen, deren verhornte Wände ihrerseits wieder aneinanderkleben, sodass ein Inhalt-Faserwerk und Eleidin, bezw. Pareleidin - hier überhaupt nicht oder nur in Spuren nachweisbar ist. Durch besondere Reagentien gelingt es jedoch, die Zellen zu isoliren und auch die Verklebung ihrer eigenen Wände zn lösen, sodass sie zu bläschenförmigen Gebilden aufquellen. Für die Beurtheilung pathologischer Processe ist es unbedingt nothwendig, diese Unterschiede im Bau der Hornschicht streng zu beachten; Re-

1) Die Buchstaben beziehen sich anf die Bezeichnung in der schematisirten Figur 9. 
sultate von Untersuchungen über das Stratum corneum von Vola manus und Planta pedis können daher niemals ohne weiteres als a u ch für die übrigen Hautstellengeltend angesehen werden und u mgekehrt.

Die Frage nach der chemischen Natur des Eleidins, bezw. des Pareleidins ist eng verknüpft mit der nach dem Fet tg ehal t der Hornschicht. Der erste, der die Behauptung aufstellte, dass das Stratum corneum Fett enthalte, war Ranvier (21). $\mathrm{L}$ a $\mathrm{n}$ gerhans (12) hatte bekanntlich gefunden, dass nach Behandlung der Haut mit Ueberosmiumsäure eine Schwarzfärbung in der Hornschicht auftritt, die nach ihm das Strat. lueid. freilassen würde und oberbalb desselben sich als ein breiter Streifen zu erkennen gäbe, eine Beobachtung, die $R$ a $n \vee i$ er bestätigen konnte. Er fand gleichfalls, dass das Strat. lucid. ungefärbt blieb, während ein breiter schwarzer Streifen unmittelbar oberhalb desselben auftrat nnd ein zweiter an der Oberfläche; daneben zeigten aber auch die Schnittränder eine intensive Schwarzfärbung. Das so entstandene Bild eines schwarzen Rahmens fübrt Ranvier darauf zurück, dass die Osmiumsäure von der freien Stückoberfläche her nach innen vordringe und so die Schwärzung verursache, dann aber ihrem weiteren Eindringen durch die gleichzeitige Fixirung des bereits passirten Gewebes selbst ein Ziel setze. Was nun den Grund der Färbung angeht, so schliesst R a nviel' aus dem Umstande, dass sie nach vorausgegangener Behandlung der Haut mit absolutem Alkohol ausbleibe, dass sie auf eine Substanz zurückzuführen sei, die in diesem letzteren Reagens löslich wäre. ${ }_{n}$ Cette substance est vraisemblablement de la graisse. Or, le stratum lucidum; en rapport direct avec des couches du revêtement épidermique encore molles, doit contenir une certaine proportion d'eau et par conséquent ne pas se laisser imbiber par les matières grasses qui infiltrent les cellules desséchées de la couche cornée. Il est donc naturel qu'il ne réduise pas l'acide osmique et qu'il soit incolore dans les préparations faites seulement au moyen de cet acide." Dieser Annahme trat Unna (30) entgegen. Nach ihm ist die Osmiumreaction der Hornschicht nicht durch das schrittweise Eindringen 
des Reagens $\%$ erklären, sondern würde auf den Consistenzverschiedenheiten derselben berthen; die von ihm hierfür angeführten Gründe sind folgende: Der basale schwarze Streifen erscheint auch dann zuerst, wenn man Schnitte von frischer Haut direct in Osmium tiberträgt; er besitzt eine konstante Breite und gerade die allerunterste Hornzellenselicht, zu der die Osmiumsäure zuerst gelangt, schwärzt sich nicht. Dagegen glaubt auch U n na, dass die Hornschicht mit Fett imprägnirt sei und dieses die Osmiumreduction bedinge; die Beobachtung, dass nach gründlicher Entfettung eine vollkommene inversion der Schwärzung eintrete, macht $\mathrm{ihm}$ jedoch wahrscheinlich, dass die Osmiumwirkung auf die Hornschicht der Einwirkung dieser Säure auf fette Körper nicht einfach gleichzusetzen sei. Koelliker (11) beschränkt sich darauf, zu bestätigen, dass nach Behandlung mit absolutem Alkohol die Schwärzung ausbleibe; er fand übrigens, dass auch das Strat. lucid. sich schwärze; über die Ursache der Reduction spricht er jedoch eine eigene Meinung nicht aus. Mit der Entdeckung des Eleidins durch Ranvier (22) war die Fettfrage in ein neues Stadium gerückt. Ranvier selbst bezeichnet es als ein ${ }_{n}$ huile essentielle", während es Liebreich (14) für ein Cholestearinfett hielt. Gegen diese beiden Behauptungen trat Buzzi (3) auf; er wies nach, dass das Eleidin weder ein ätherisches Oel, noch Lanolin sei und erklärte dasselbe für ein fettes Oel, ein Glycerinfett, vorzüglich wegen der Osmiumreaction der Tropfen. $\mathrm{R}$ abl (20) bestreitet die Angaben $\mathrm{B} \mathrm{u} \mathrm{z} \mathrm{z} \mathrm{i's;} \mathrm{die} \mathrm{Osmiumreduction} \mathrm{der} \mathrm{Hornschicht} \mathrm{führt} \mathrm{dieser} \mathrm{Autor}$ gleichfalls auf den Fettgehalt derselben zurück, jedoch findet er, dass das Eleidin bei Behandlung frischer Hautschnitte mit Osmium keine Schwarzfärbung annimmt, also auch kein Glycerinfett sein könne. Doch hält er es für möglich, dass das weitere Umwandlungsprodukt des Eleidins ein Fett ist, oder wenigstens ein solches aus demselben abgespalten werden kann. Aus dem Auftreten blauer und rother Zellen bei Behandlung mit polychromsaurem Methylenblau schliesst R a u s c h (25), dass diese Färbungsdifferenzen durch den Gehalt der Zellen an Fett und zwar wahrscheinlich an verschiedenen Fetten in Bezug auf die Nuance beeinflusst werden, trotzdem nach Behandlung mit kochendem Aether diese Verschiedenheiten sich nicht ansgeglichen hatten. Die Frage nach dem Fettgehalt erhielt weiterhin einen neuen Anstoss 
durch die auffälligen Befunde Unna's, die er mit seiner secundären Osmirung $(31,32)$ gefunden hatte. Er fixirte Hautstückchen in der bereits oben angegebenen Mischung, bettete sie dann auf dem gewöhnlichen Wege in Celloidin ein und behandelte nachher die Schnitte mit einer Osmiumalaunlösung $(1,0 \mathrm{a} \overline{\mathrm{a}})$ in der Wärme. Auf diesem Wege erhielt er eine ausgedehnte Schwarzfärbung; darnach würden reichliche Fettmassen - ich erwähne hier nur die für unsere Frage speciell in Betracht kommenden Zellen - sowohl im Strat. germinat. als auch im Strat. corn. nachweisbar sein; das Fett läge in diesen Schichten in den Kernhöhlen und Lymphspalten. Jedoch macht $U$ nn a eine Einschränkung; die Fettbefunde sollen nämlich nicht constant sein, am reichlichsten fänden sie sich in der Hant der Fusssohle älterer Individuen von $40-70$ Jahren, und wie ich einer liebenswürdigen Privatmittheilung Herrn U n in a's entnehme, auch lier nur unter zehn Fällen viermal in höherem Grade, riermal in geringerem, zweimal nur in Spuren und zweimal inberhaupt nicht. In der Deutung der schwarzen Bildungen als Fett ist jedoch $\mathrm{Unn}$ a zum Theil scharfem Widerspruch begegnet. ML e res (vgl. 31) hat dieselben für Niederschläge erklärt; $\mathrm{T} \ddot{o} \mathrm{rök}$ (28) behauptet sogar, dass ähnliche von $\mathrm{Unn}$ a beim Ekzema seborrhoicum beschriebene, mit Osmium geschwäryte Tropfen Luftblasen wären. Gegentber diesen Einwänden hat Lö w e n b a c b (15) die Befunde $\mathrm{Un} n$ a's bestätigen können. Er fixirte Fusssoblenbaut nach Un $n$ a oder in Fle mm i $n$ g'scher Lösung und färbte dann mit Sudan III; hierbei fand er die Kernhöhlen der Hornschicht mit rothen Fettmassen gefüllt, ebenso Fett an allen von U $\mathrm{n}$ a beschriebenen Stellen; aus seinen Angaben verdient jedoch hervorgehoben zu werden, dass er an 41 Fusssohlen elfmal einen positiven Befund hatte, dagegen konnte er in den untersuchten 25 Fällen von frischer Haut weder mit Sudan noch mit Osmium Fett nachweisen. S a ta (26) pruffte gleichfalls an einem reichlichen Material die $\mathrm{U} n \mathrm{n}$ a'schen Angaben, er fand jedoch mit der secundären Osmirung nienals Fett in den Kernböhlen und Lymphspalten des Strat. corn., dagegen wohl in Form kleiner Körnchen in den tiefen Schichten des Strat. germin., wo ähnliche Beobachtungen bereits von Le d e r $m$ a $n(13)$ und Wents c he r (34) gemacht waren; bei einer Nachuntersuchung mit Sudan III waren jedoch rothe Tröpfchen in jenen Zellen nicht 
nachweisbar; er schliesst daraus, dass die durch Osmium dargestellten Granula jedenfalls nicht völlig ans Fett bestünden. Endlich neigt $\mathrm{M} \mathrm{e} \mathrm{r} \mathrm{k}$ (16) zu der Annahme, dass U n n a's Fettbefunde richts anderes wären als Osminmreductionen, veranlasst durch die in der Fixirungsflüssigkeit benutzte Gerbsäure. An Gefrierschnitten durch die Sohlenhaut war nach Merk's Beobachtungen nach einer halben Stunde weder im Strat. germin. noch im Strat. corn. oder in den Schweissdrüsen irgendwelche auf Fett zu beziehende Farbenänderung nachweisbar; an behaarter Haut schwär\%te sich das Strat. corn. nur in der Umgegend der Haare in einel oberflächlichen dünnen Zone. In den tiefen Epidermiszellen traten dagegen gleichfalls schwarz gefärbte Granula auf, jedoch vollzog sich hier die Osmiumreduction später als all den typischen Fettzellen des Unterbautbindegewebes.

Soweit in der Hauptsache die Literatur inber die Fettfrage. Zum Zweck einer kritischen Betrachtung all dieser zum grossen Theil sich widersprechenden Angaben mussten die angeführten Versuche wiederholt werden; ihre Resultate will ich zunächst mittheilen. Zu meinen Untersuchungen wählte ich aus später anzufübrenden Gründen Sohlenhaut; die noch warmen Leichen entnommen wurde; dabei trug ich die Haut sorgfältig mit dem Rasirmesser ab, um kein Cutisfett, wenn nicht besonders gewünscht, in das Präparat zu bekommen. Bringt man derartige Haut in eine $1 \%$ Osmiumsäurelösung auf ca. 5 Stunden, wäscht dann in Wasser aus und bettet in Paraffin oder in Celloidin ein, so erscheint das ganze Strat. germinat. und granul. grünlich gefärbt, die Keratohyalinschollen des letzteren haben gleichfalls eine grine Farbe angenommen, das Strat. lucidum, in dem das Eleidin in fester Form erscheint, ist olivengrün. Von dem eigentlichen Strat. corn. sind die Seitenränder des Schnittes geschwäl"zt, eluenso die oberflächliche Schicht, während ein basaler Streifen sich eben durch eine dunkle Linie anzuzeigen beginnt. Ich möchte gleich hier hervorheben, dass auf dünnen Schnitten die Farbe der os. mirten Zellen deutlich schwarıgrün ist und sich dadurch von osmirtem Fett immerhin im Aussehen unterscheidet. Hat die Osmiumsäure längere Zeit, etwa 12 Stunden, eingewirkt, so zeigt sich, dass sowohl der basale als der oberflächliche Streifen an Breite zugenommen haben, ebenso die Seitenränder. Diesen 
basalen Streifen rechnet U n n a (29) zum Strat. lucid., das er deswegen in zwei Unterabtheilungen $-4 \mathrm{a}$ und $4 \mathrm{~b}$ - eintheilt; während $4 \mathrm{a}$ sich durch Osmium schwärzen soll, würde $4 \mathrm{~b}$ ungefärbt. bleiben; infolgedessen nimmt er für das Strat lucid. sechs Zellreihen an. Dem kann ich jedoch nicht beistimmen. D a s Strat. lucid. umfasst nicht mehr als 2-3 Zelllagen; man kann sich davon leicht aberzeugen, wenn man einen diunnen Schnitt frischer oder wenig in Alkohol gehärteter Haut mit Osmium behandelt; wälırend dabei das übrige Strat. corn. eine dunklere Farbe annimmt, behält das Strat. lucid. lange seinen stark lichtbrechenden Charakter, der sich jedoch nur auf 2-3 Reihen heschränkt. Auch ist übrigens das flüssige Eleidin nur in den 2-3 basalen Zelllagen nachweisbar, und endlich hat auch der Entdecker der Schicht, $\mathrm{O} \mathrm{ehl}$, nur 2-4 Reihen angenommen, er sagt ausdrücklich (19): Le cellule dello strato lucido trovansi disposte a non oltre due, tre o quatro ordini. In seiner Abhandlung erscheint allerdings das Strat. lucid. (auf Taf. II Fig. 17) ziemlich breit; dies aber hat darin seinen Grund, dass die Zellen jenes Schnittes durch die Behandlung - bollita nella potassa stark gequollen sind. Ein vorzügliches Mittel in osmirten Präparaten das Strat. lucid. darzustellen, ist die Färbung mit Pfitžnerschem Safranin und nachheriger Differenzirung in schwach angesäuertem absolutem Alkobol. Wie die Fig. 10 wiedergiebt, färben sich dabei nicht nur die Keratohyalingranula ausserordentlich scharf und intensiv roth $(s g r)$, sondern anch die nicht geschwärzten Hornzellen, die nach innen von dem basalen Osmiumstreifen $(b)$ nur 2-3 Zellreihen ausmachen $(s l)$. Demnach besteht keine Veranlassung, das Strat. lucid. in zwei Unterabtheilungen zu zerlegen.

Bleiben die Hauptsticke 24 Stunden lang in der Osmiumsäure, so schreitet die Schwärzung immer mehr von den Rändern her gegen die Mitte zu fort, sodass sich an einzelnen Stellen die ganze Hornschicht gesehwärzt erweist, mit Ausnahme des Strat. lucid., an dem ich hier nur eine tief dunkelgrüne Färbung beobachten konnte. An einem derartigen Präparate war ich in der Lage, an einer Zelle des Strat. granulos. eine eigenthümliche, bisher meines Wissens nicht beobachtete Erscheinung zu constatiren, auf die ich kurz eingehen möchte. Die Zelle (Fig. 11) liess neben einem deutlichen etwas geschrumpften Kern $(n)$ und 
zahlreichen kleinen mehr hellgrün gefärbten Keratohyalingranula $(k)$ eine grosse dunkelgriin tingirte runde Masse (e) erkennen, die sofort den Eindruck eines grossen Tropfens macht. Ich habe daraufhin meine sämmtlichen Präparate durchmustert und nur noch einmal an einem in Formol fixirten und mit Heidenhain'schem Hämatoxylin gefärbten Schnitt dieselbe Beobachtung machen können; dort war dieser Tropfen intensiv schwarzblau gefärbt. In beiden Fällen entsprach also die Färbung genau der des Eleidins innerhalb des Strat. lucid.; nimmt man dazu die ausgesprochene runde Form der Masse mit den glatten Conturen, die so ohne weiteres als Tropfen imponirt, so kann es keinem Zweifel unterliegen, dass wir es hier thatsächlich mit Ele id in zu thun haben, das im frischen Zustande eine Flüssigkeitsvacnole in der Zelle gebildet hat, die dann, durch die Behandlung fest geworden, auf dem Schnitt als eine Scheibe erscheint. Für die Ansicht, dass das Eleidin durch Verflussigung des Keratohyalins entstunde, bietet diese Beobachtung einen weiteren Beweis; man kann sich denken, dass hier aus irgend welchem Grunde nahe beisammen liegende Keratohyalinkörner vorzeitig verftüssigt wurden und zusammenfliessend zur Entstehung einer Eleid in vacu ol e in der Strat. granul.-Zelle Anlass gegeben haben. Es ist übrigens interessant, dass $\mathrm{B} \mathrm{u} z$ z $\mathrm{i}$ (4) bei Warzen häufig Keratohyalin neben Eleidin in denselben Zellen angetroffen hat.

Um nun wieder zu der Deutung der Osmiumreaction der Hornschicht zurückzukehren, so schliesse ich mich hierin völlig den Autoren an (Langerhans, Ranvier, Rabl), welche die eigenthümliche Rahmenbildung auf die Art des Eindringens des Reagens zurückfubren. Trotzdem auch ich die Meinung vertrete, dass Spannungsverschiedenheiten in der Schichtung des Strat. corn. bestehen und dass diese von z. T. bestimmendem Einfluss auf die Fixation sind, glaube ich doch, dass man nicht berechtigt ist, diese für das Osmiumbild verantwortlich $\mathrm{zu}$ machen. Ich kann zunächst die Angabe, dass bei frischen Hautschnitten der basale Streifen zuerst auftreten soll, nicht bestätigen; die dagegen gern zngegebene Thatsache, dass er an fixirtem Material trotz fortgeschrittener Osmiumwirkung eine gewisse Breitenconstanz zeigt, fuhre ich darauf zurtick, dass von der Cutisseite her durch das vorliegende und bereits fixirte Strat. germinat. und granul. das Eindringen des Reagens bedeutend mehr erschwert ist, als Archiv f. mikrosk. Anat. Bd. 57 
dies von der freien Oberfläche her der Fall ist. Dass ferner das Strat. lucid. trotz seiner lcichten Zugänglichkeit sich nicht schwärzt, hat seinen Grund eben in chemischen Differenzen des Eleidins gegenüber dem Pareleidin, wovon noch die Rede sein wird. Der Einwand Unna's, dass sowohl der basale wie der obere Streifen sich immer schärfer abgrenzen, während die Schwarzfärbung des Randes ungleicbmässig nach innen vorspringe, findet seine Erledigung in der Anorduung der Zellreihen, die ja im grossen und ganzen parallel übereinanderliegen, sodass der grösste Zelldurchmesser stets auch parallel sich mit der Eintrittsebene des Reagens befindet; dadurch wird dieses, vou oben nach unten und umgekehrt fortschreitend, eine Zellreihe nach der andern ergreifen, also stets eine ziemlich scharfe horizontale Abgrenzung erkennen lassen, während an den Seitenründern, wo die Zellen senkrecht zu der Eintrittsebene angeordnet sind, die Enden der einzelnen fixirten und geschwärzten Zellen je nach ihrer Grösse und Lage bald weiter, bald kürzer nach innen vorspringen müssen. So entsteht hier eine ungleichmässige Abgren\%ung der Eintrittslinie. Die Beobachtung Rabl's, dass der basale Streifen von den Seitenrändern her nach innen vorzurticken scheint, kann ich an fixirtem Material bestätigen; da der Streifen etwa dem Strat. relaxatum ( $s r$, Fig. 9) entspricht, das locker geschichtet ist, wird sich hier die eindringende Flüssigkeit rascher verbreiten können, namentlich dann, wenı diese Lage durch den Seitenschnitt eröffnet wurde. Dies spricht ja nun allerdings in gewissem Grade für die Unnasche Annahme, doch neige ich aus den obenangeführten Grïnden dazu, das Osmiumbild als eine Folge des bekannten Durchtränkungsmodus dieses Reagensanzusehen; im Einzelnen mag das Eindringen durch die Schichtung etwas erleichtert oder erschwert werden.

Wie verbält sich nun Haut, die vorber mit absolutem Al k o h o l behandelt war, gegenüber der Einwirkung der Osmiumsäure? Zur Prufung dieser Frage wurden kleine Stückchen Sohlenhaut auf 24 Stunden direct in absoluten Alkohol gebracht, dann wher Nacht in fliessendem Wasser ausgewaschen, anf 24 Stunden in eine $1 \%$ Osmiumlösung eingelegt und weiterhin in Paraffin eingebettet. Auch jetzt zeigte sich eine vollständige Schwarzfärbung und zwar des ganzen Strat. corn. mit Ausnahme des Strat. lucid. Jedoch fiel mir dabei eine Besonderheit 
auf, während frisch osmirte Haut bei ibrer Entnahme aus der Fixirungsflüssigkeit völlig geschwärzt war, trat bei der erst mit absolutem Alkohol behandelten diese äussere Schwarzfärbung erst bein Auswaschen und nach der Ueberfithrung in Alkokol ein. Es erinnert dies an die Beobachtung $S$ t tarke's (27), dass die Osmiumreduction bei einzelnen Fetten erst secundär in Alkohol eintritt (Alkohol-Osmium-Reduction). Zunächst möchte ich noch erwähnen, dass es mir ebenso wenig wie anderen \%. B. M erk gelungen ist, eine Nichwar\%ärbung des Ntrat. corn. zu erhalten, wenn ich frische sichnitte, entweder mit dem Rasirmesser oder mit dem Gefiriermikrotom angefertigt, in die Osmiumlösung brachte. Nach längerer, mehrere Stunden dauernder Einwirkung des Reagens wurde zwar die Hornschicht in toto grün, niemals aber beobachtete ich eine Schwärung wie bei der Stückosmirung.

Was nun die secundäre Osmirung angeht, so konnte ich mich trotz genauester Befolgung der Un n a'schen Angaben von dem Vorhandensein schwarzer und als Fett gedenteter Körper nicht überzeugen. Zwar nahmen die Schnitte die Osmiumsäure sehr schön an, d. h. sie zeigten, wie Fig. 8, deutlich die charakteristische grïne Osminmfarbe; aber weder in den Kernhöhlen noch sonstwo erhielt ich eine Nchwarzfärbung, mit Ausnahme zweier Fälle, in denen schwarze Körner in den tiefen Lagen des Strat. germinativ, auftraten (Fig. $12 \mathrm{~g}$ ). Diese negativen Resultate, die trotz wiederholter Versuche constant waren, brachten mich daranf, einmal nachzuprüfen, ob denn überhaupt mit dem Unnaschen Verfahren sich wirkliches Fett fixiren und secundär osmiren lasse. Ich nahm dazu Fett aus der Nierenkapsel des Kaninchens und aus dem Unterhautbindegewebe des Menschen, brachte es auf 24 Stunden in die Unna'sche Mischung, wusch in fliessendem Wasser aus, bettete in Celloidin ein und behandelte die gewonnenen Schnitte sofort mit der Alaun-Osmiumlösung. Das Resultat war überraschend: ein leeres Maschenwerk ohne jede Spur von Fett in den Fettzellen. Fixirt man gar in der Wärme, wie es Unna für die Haut empfiehlt, so beachtet man, dass das Fett ohne weiteres austritt und in Form von Augen auf der Fixirungsflüssigkeit herumschwimmt. Auf Grund dieser Thatsache bestreite ich entschiedendie Fettnatur der nach der Unna'schen Methode zur Darstellung gebrachten $\mathrm{Schwär}$ ungen, gegen die übrigens noch andere Momente 
sprechen. Wenn nüılich thatsächlich die Kernhöblen der Hornzellen Fett enthielten, warum sollte sich dann dieses Fett nicht ebenso gut dureh die primäre Osmirung darstellen lassen? In dem Strat. disjunctum ( $s d$, Fig. 9) lassen die Zellen bei Anwendung dieser Methode ein schwarzgrünes oder schwarzbraunes Faserwerk $(f)$ erkennen (Fig. 13) mit einer centralen Kernhöhle (c), in der ich niemals einen schwarzen Körper nachweisen konnte; dass ubrigens an diesen Zellen das Fasernetz so deutlich hervortritt, hat seinen Grund darin, dass hier das Pareleidin, woftir ich bereits mehrere Beobachtungen anführte, sich im Zustande der Eintrocknung befindet und so seinen homogenen, das Netz verdeckenden Bal eingebüsst hat. Weiterhin muss auffallen, dass an Hornzellen, die in Osminmsäure macerirt und isolirt wurden, keine schwarzfärbung beobachtet werden kann, sondern nur eine grüne Tinction der Fasern; ebensowenig ist etwas von einem schwarzen Körper in der Kernhöhle zu beobachten. Ferner wäre noch zu erwähnen, dass sich auch an frischer mit dem Rasirmesser oder mit dem Gefriermikrotom geschnittener Haut bei Bebandlung mit. Osmium dieselbe Thatsache constatiren lässt; insbesondere tritt bei Färbung mit Sudan III keine rothe Masse im Zellinnern auf. Alle diese Befunde sprechen also gegen die Fettnatur der Unna'schen Schwärzungen. Mich in speculationen einzulassen, wie dann die von $\mathrm{Unn}$ a dargestellten Bilder zu deuten sind, liegt kein Grund vor; ich halte aber mit Merk für das wahrscheinlichste, dass wir es dabei mit einer Gerbsäure-OsmiumReduction zu thun haben; die Fixirungsflussigkeit entlualt nämlich $1 \%$ Gerbsäure; möglicherweise bängt es von dem Auswaschen ab, ob die Tanninsäure melr oder weniger reichlich im Gewebe zurückgehalten wird und dann die Osminmsäure stärker oder schwächer reducirt; dafür scheint zu sprechen, dass die Schwärzungen nach Unna hauptsächlich in Höhlen oder Kanäleu sich finden (Kernhöhlen, Schweissdrüsengang, Blutgefässe, Lymphspalten). Aber selbst das Unmögliche zugegeben, es handle sich um Fett, so spricht doch die Seltenheit des Vorkommens dagegen, dass wir es mit einem normalen Befund zu thun haben; nach Unna's eigenen Angaben und nach den Untersuchungen von Löwenbach (10̄) würde es sich nämlich nur bei Personen zwischen dem 40. und 70. Lebensjahre finden und auch hier nur in etwa $40 \%$ der Fälle. 
Was nun die schwarzen Körnchen in den tiefen Lagen des Strat. germinat. betrifft, so wurde deren Vorkommen von Sata (26) bestätigt, und auch ich habe derartige Bilder, wie oben erwälnnt, gesehen (Fig. 12). Die von mir beobachteten, mit secundärer Osmirung dargestellten Körnchen fanden sich allerdings nur auf eine bestimmte Zone beschränkt, nie in der Hornschicht noch auch in der Cutis. Dagegen zeigrten sie in ihrer Lage zu den Zellen absolut nichts charakteristisches; bald liatte ich den Eindruck, dass sie auf der Zelle lagen, bald schienen sie in das Protoplasma selbst eingebettet $z u$ sein, einigemal auch fand ich sie im Kern, ja sogar im Intercellularraum. Ihre Form ist nichts weniger als rund oder überhaupt regelmässig, sie zeigen vielmehr am Rande Zacken und Ausbuchtungen, sodass ich sie trotz der Beschränkung ihres Vorkommens auf eine bestimmte Zone für Osmiumniederschläge halten möchte. Selbstverständlich kann ich in diesem Falle nur meine eigenen Präparate beurtheilen, da ich die Befunde von Sata und Wentscher (34) nur aus den Abbildungen kenne. Gegen die Fettnatur spricht durchaus hier, dass die Unna'sche Methode das Fett iberhaupt nicht $\% 111^{\circ}$ Darstellung bringt und ferner ihr Verhalten dem Sudan gegentiber, wobei sie nach Sat a viel kleiner als nach Osmiumbehandlung sein sollen; ich selbst habe sie übrigens mit sudan nicht nachweisen können. Thatsächlich hat sich auch schon Lederm an n (13), der sich am ausführlichsten mit diesen Körnchen beschäftigt, uber ihre Fettuatur mit grosser Zurtickhaltung ausgesprochen; mich wundert nur, dass nicht an die Möglichkeit gedacht wurde, dass die Köruer Glykogen sein könnten, das sich ja in der Epidermis nicht selten findet.

Kommen also auch die Unna'schen Befunde für den Fettgehalt des Strat. corn. nicht in Betracht, so bleibt doch die Thatsache seiner Schwärzung bei Osmiumfixirung bestehen. Da wir nun ohne weiteres anzunehmen geneigt sind, diese Reduction auf Fett zurtackzufithren, so ist nicht $z u$ verwundern, dass allgemein die Schwärzung der Hornschicht dementsprechend beurtheilt wird. Es ist dabei aber in hohem Grade auffallend, dass von den meisten Autoren sich niemand mit Bestimmtheit darïber ausspricht, woher denn eigentlich dieses Fett der. Hornzelle stammen wiurde. Die Quelle kann zweierlei Art sein: entweder gelangt 
es von aussen her in die Zelle hinein oder aber es entsteht in der Zelle selbst.

Der ersteren Annahme scheint Ranvier (21) zuzuneigen, da

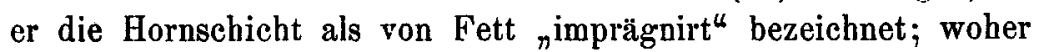
aber dieses Fett kommt, wird ron ihm nicht erwähnt. Gelangt es von aussen her in die Hornzelle, so können nur die $\mathrm{Haut-}$ $\mathrm{d} \mathbf{r} \mathfrak{u}$ se $\mathbf{n}$ dabei in Betracht kommen. Wir mässteń uns dann vorstellen, dass diese Drüsen Fett produciren, das dann in flissiger Form durch die Hornmembran der Zelle hindurch in diese hineindringen wiirde. Solche Fett producirende Organe sind die $\mathrm{T}$ algd $\mathrm{r}$ use $\mathrm{n}$, durch sie kann also Fett in das Strat. corn. ausgeschieden werden. Dabei ist aber zu bedenken, dass sich diese Drüsen weder an der Vola manus noch an der Plant a pedis finden, während man doch gerade an dieser Haut den charakteristischen Osmiumbefund erbält. An der übrigen Haut können dagegen jene Organe eine Fettimprägnation verursachen, und der Befund scheint thatsächlich dafür zu sprechen. Ich habe Hodensackhaut, die sich ja durch den Gehalt an besonders grossen Talgdrüsen auszeichnet, daranfhin untersucht und konnte hierbei constatiren, dass die Schwarıfärbung nicht die ganze Hornschicht umfasst, sondern sich auf einzelne mehr oder weniger ausgedehnte Stellen beschränkt. Die geschwärzten Partien fanden sich im allgemeinen vorherrschend in der Umgebung der Talgdrtisenmundung auf der Oberfläche, sodass hier thatsächlich eine Beziehung zwischen Hautschwärzung und Fettausscheidung der Drüsen zu bestehen scheint. Immerhin aber ist diese Fettquelle wegen des Fehlens der Drüsen für die Hornschicht von Vola manus und Planta pedis auszuschliessen; da an die Möglichkeit gedacht werden muss, dass die Haut der Handfläche durch $\mathrm{Be}$ rührung behaarter Körperstellen eingefettet werden kann, wählte ich, um ganz sicher za gehen, für meine Untersuchungen Sohlenhant, wobei diese Quelle kaum in Betracht kommen kann.

Hier könnten also nur die $\mathrm{S}$ chweis $\mathbf{s}$ drü $\mathrm{sen}$ das Fett liefern. Bekanntlich wird ja für diese Drtisen von einzelnen Autoren eine Fettsecretion angenommen; ich will auf diese Frage hier nicht näher eingehen und möchte nur erwähnen, dass meine Beobachtungen nicht zu gunsten jener Annabme sprechen, da es mir weder mit der primären Osmirung noch mit der Sudanfärbang von Gefrierschnitten jemals geglückt ist, in den Knäueln oder den 
Ausfïhrgängen Fett nachzuweisen. Es wäre aber immerhin denkbar, dass ich, wenn wir mit $\mathrm{K}$ o ell i ke r (11) annehmen, dass die Fettsecretion möglicher Weise mit der des Schweisses alternirt, gerade immer das Unglück gehabt habe, in fettfreien Momenten zu fixiren. Deswegen sei die Fettsecretion zugegeben, und es würde sich demnach fragen, ob die Schweissdrïsen das Fett liefern, welches die Hornschicht imprägniren würde. Wäre dies der Fall, so wïrde also das in den Knäueln producirte Fett die Ausführgänge passiren, auf die Oberfäche des Strat. corn. ausgeschieden und sich dort ausbreiten. Da nun innerhalb der Hornschicht der Ausführgang einer eigenen Wandung entbehrt, könnte das Fett noch in den Intercellularräumen vordringen, dieselben umspïlen und dann schliesslich in das Innere derselben gelangen. Dazu wäre aber Voraussetzung, dass es sich in einem flüssigen Zustande befindet; ob nun die Temperatur der Hornschicht noch so hoch ist, um dies $\mathrm{zu}$ gestatten, erscheint mir mehr als fraglich. Aber ich will selbst den Fall setzen, dass dem so wäre, dann hätten wir uns vor\%ustellen, dass das Fett entweder direct von den Ausführgängen der Sehweissdrüsen aus nach allen Seiten die Hornschicht imbibirt oder aber von der Oberfläche her, d. $h$. von der Gangmündung aus, nach unten durchsickert und die Hornzellen durchtränkt. Stimmt nun damit das Osmiumbild? Wenn wir die geschwärzten Partien betrachten, so stellt sich ohne weiteres heraus, dass alle Stellen gleich intensiv gefärbt sind, die Schwärzung also nicht etwa in der Nähe des Ganges oder an der Oberfläche stärker ist und von da nach unten oder irgend einer Seite zu abnimmt; ich habe bereits darauf hingewiesen, dass die Zellreihen des Strat. disjunctum (Fig. 13) sogar weniger intensiv gefärbt sind, als die darunter gelegenen. Die Osmirung steht also nicht im Verhältnis zu der Entfernung der Zellen von den Schweissdrüsengängen. Da ferner feststeht, dass die Schwärzung plötzlich eintritt, sobald die Zelle bei ihrem Wege nach der Oberfläche zu eine bestimmte Höhenzone, nämlich das Strat. lucid., überschritten hat (Fig. 14), so müsste sich also hier sofort die Zelle mit Fett imprägniren: das könnte natürlich nur dann der Fall seiu, wenn die ganze Hornschicht beständig, wenn ich mich so ausdrücken darf, "unter Fett gesetzt" wäre; für eine solche Annahme spricht aber absolut nichts. Ueberall also den besten Fall gesetrt, dass die Fettsecretion der Schweissdrusen 
tibor allen Zweifel erhaben wäre, dass ferner die Tempe. ratur es gestatten würde, dass das Fett in flüssigem $\mathrm{Zu}$ stande die Holnmembran passirt und die Zelle imprägnirt, so spricht immer noch die gleichmässige und vondem Verlauf der Drüsengänge völlig unabhängige Schwärzung gegen diese Erklärang. Demnach besteht aller Grund, immer angenommen, die Osmiumreaction der Hornschicht ron Vola manus und Planta pedis beruht auf Fett, eine Durchtränkung der Zellen durch das Secret der Talg-oder Schweissdrüsen a us u s chliessen.

Es bliebe also noch die Möglichkeit zu erörtern, dass das Fett in den Zellen selbst entsteht. Da die Schwarzfärbung der Zelle eine gleichmässig homogene ist und die ganze Zelle erfullt (sr Fig. 14), so muisste ihr die Substanz zu Grunde liegen, die sich ebenso verhält, das wäre also das Eleidin, bezw. das P a releidin. Das Eleidin ist nun bereits wiederholt seiner physikalischen und chemischen Eigenschaften wegen für Fett gehalten worden, besonders weil es sich in Osmium schwärzen soll. Ich habe bereits darauf hingewiesen, dass diese Schwärzung von vielen bestritten wird, und auch ich kounte sie nicht constatiren. Das Strat. lucid. blieb auch bei lang dauernder Osmiumeinwirkung ungeschwärzt (sl, Fig. 10 und 14). Aber auch diejenigen Autoren, die eine Schwärzung beobachtet haben wollen, geben übereinstimmend an, dass sie erst nach selır langer Fixirung eintrat, ein Moment, das die Fettnatur des Körpers auss chliesst. Wie ich nämlich den Ausführungen Munk's (18) entnehme, bängt mit dem Grade der Flüssigkeit eines Oeles oder Fettes auch der Gehali. an verschiedenen Fetten zusammen: der flussige Zustand des Eleidins wïrde darnach voraussetzen, dass es besonders reich an Oclsäure, bezw. Olein, ist, Palmitin und Stearin dagegen in nur sehr geringer Menge enthalten würde. Nun haben aber sowohl A l t m a n n (1) als auch $\mathrm{H}$ a nd we r ck (10) nachgewiesen, dass eine rasche Reduction der Osmiumsäure nur durch den Gehalt eines Fettes an Oelsäure, bezw. Olein, bedingt wird, wäbrend die beiden anderen Fettsäuren nicht im Stande waren, Osmium zu reduciren: nach $\mathrm{Handwerck}$ ist eine intensive Schwärzung bereits nach 1-2 Stunden eingetreten. Diese $\mathrm{Thatsachen}$ sprechen mit Sicherheit gegendieFettnaturdes 
Ele id ins. Wie verhält es sich nun mit dem Pareleidin? Sobald das Eleidin in den festeren Aggregatzustand übergeht, also jenseits des Strat. lucid., tritt die Schwärzung ein: es wäre also an die Möglichkeit $z u$ denken, dass das Eleidin sich in Fett umwandelt. Allein nach allem, was wir über die Umwandlung eines Zellbestandtheiles in Fett wissen, folgt, dass der Uebergang ein allmählicher ist, indem zuerst einzelne Fetttröpfchen auftreten, die schliesslich mit einander confluirend eine einheitliche Fettmasse bilden. Wie aber aus Fig. 14 (sr) hervorgeht, ist von solchen Tröpfchen nichts zu sehen; die Schwärzung ist sofort eine homogene und die ganze Zelle ist davon gleichmässig erfüllt. Wenn man Haut in Alkohol fixirt, in Celloidin einbettet und die Schnitte dann mit Osmium behandelt, so bleibt die Schwärzung aus. Wäre hier Fett gelöst worden, so müssten, etwaigen kleinen Fetttröpfchen entsprechend, Vacuolen in den Zellen vorhanden sein, oder aber der gesammte homogene Zellinhalt fehlen. Beides aber ist, wie ein Blick auf die Fig. 15 zeigt, nicht der Fall; diese Zellen unterscheiden sich nur dadurch von den osmirten, dass sie grün gefärbt sind; Anzeichen dafür, dass etwas extrahirt wurde, bestehen, trotz des Ausbleibens der Osmiumwirkung infolge der Behandlung, nicht. Alle diese Beobachtungen sprechen dafür, dass das Eleidin nicht einer fettigen Metamorphose unterliegt, also auch das Pareleidin kein Fet sein kann.

Dass die Osmiumreduction der Hornschicht thatsächlich nicht auf einen Fettcharakter des Pareleidins zurückzuführen ist, wird zur Sicherheit durch den Entfettungsversuch. Zu diesem Zwecke wurde frische Sohlenhaut 24 Stunden lang im SoxhletApparat mit kochendem Aether behandelt ${ }^{1}$, dann anf ebenso lange Zeit in eine $1 \%$ Osmiumsäurelösung uibergeführt, ausgewaschen and in Celloidin eingebettet. Der Erfolg war auch hier ebenso wie bei dem oben angefürten Alkoholversuch e in e deutliche Schwarzfärbung der Hornschicht, nur mit dem Cnterschiede, dass auf dünnen Schnitten die Schwärzung einen Stich ins Braune zeigte statt ins Grïne, wie bei frischer

1) Herr Dr. Hajo Bruns, Assistent am bacteriologisch-hygienischen Institut, hatte die Liebenswürdigkeit, die Fettextraction vorzunehmen, wofür ich ihm auch hier meinen Dank ausspreche. 
Haut, eine Differenz, die sie tubrigens mit dem Strat. germinativ. theilte. Eine Umkehrung der Schwärzung, wie sie Unn a beschreibt, konnte ich dagegen nicht beobachten. Was nun noch die Sudanreaction frischer mit dem Rasirmesser oder mit dem Gefriermikrotom geschnittener Haut betrifft, so nimmt die Hornschicht stellenweise die Sudanfärbung überhaupt nicht an, an anderen Orten zeigt sie dafür eine hellrosa Färbung, die auch auf das Strat. germinat. übergreift. Wie aus den Angaben Daddi's (5) hervorgeht und wie man sich stets auch selbst überzeugen kann, wenn sich an dem Hautschnitt noch fetthaltige Cutis findet, ist nur eine orangerothe Färbuag für Fett charakteristisch, nicht aber eine Hellrosafarbe. Aus dem ersteren Versuche geht nun hervor, dass eine $S \mathrm{chwar}$ färbung des Pareleidins durch Osmium selbst dann noch eintritt, wenn alles Fett extrahirt ist; aus dem zweiten folgt, dass das Pareleidin keine Fettreaction mit Sudan giebt. Demnach ist das Pareleidin kein Fett, dabei aber doch im Stande, Osmium za reduciren; das letztere erscheint nicht mehr anffallig, da es Beobachtungen genug giebt, die beweisen, dass auch andere Körper ausser Fett fähig sind, die Ueberosmiumsäure zu reduciren; ihnen wird das Pareleidin anzureihen sein.

Zusammenfassend können wir also sagen:

1) Die Schwarzfärbung der Hornzellen von Vola manus und Planta pedis dureb die 0 smiamsäure ist nicht a u eine Fettimprägnation des Stratum corneum von aussen her, d. h. durch das Secret der Talg-

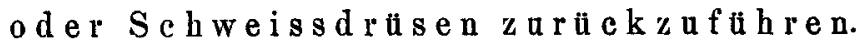

2) Die Annahme, dass das Fett in den Zellen selbstentsteht and mit dem Eleidin, bezw. Pareleidin identisch ist, oder aus ihm abgeschieder wird, ist gleichfalls z u verwerfen.

3) Das Pareleidin, nicht auch das Eleidin, besitzt die Eigenschaft, die Osmiumsäure zu reducieren, jedoch erst nach längerer Einwirkung des Reagens als dies bei wirklichem Fettder Fallist; 
4) Diemit secundärer Osmirungerbaltenen Schwärzungen beruhen nicht auf Fett, sondern sind sehr wabrscheinlich $\mathrm{Ni}$ ederschläge.

5) Anden beharten Hautstellen ist die Osmiumreduction wahrscheinlich auf eine Fettimprägnation der Hornsebicht durch das Secret der Talgdräsen zurückzuführen.

1. October 1900.

\section{Literatur - Verzeichniss.}

1. Altmann, Die Elementarorganismen und ihre Bezichungen zu den Zellen. Leipzig 1894.

2. Blaschko, Beiträge zur Anatomie der Oberhaut. Arch. f. mikr. Anat. Bd. 30.1887.

3. Buz\%i, Keratohyalin und Eleidin. Monatsh. f. pract. Dertnatolog. Bd. 8.1889.

4. Derselbe, Ueber Eleidin. Ebenda Bd, 23. 1896.

5. Da ddi, Nouvelle méthode pour colorer la graisse dans les tissus. Arch. ital. de biolog. Bd. 26.1896.

6. Dreysel und Opplex, Beiträge zur Kenntuiss des Eleidins in normaler und pathologisch veränderter Haut. Arch. f. Dermatol. und Syphil. Bd. 30. 1895.

7. Ebner v., Untersuchungen über die Ursachen der Anisotropie organisirter Substanzen. 1882.

8. Grosse, Ueber Keratohyalin und Eleidin und ihre Beziehungen zum Verhornungsprocess. Inaug.-Dissert. König:sberg 1892.

9. Frickenhaus, Zur Technik der Eleidindarstellung. Monatsh. f. pract. Dermatol. Bd. 23.1896.

10. H a nd werck, Bejträge zur Kenntniss vom Verhalten der Fettkörper zur Osmiumsäure und zu Sudan. Zeitschr. f. wissensch. Mikrosk. Bd. 15. 1898.

11. Koelliker, Handbuch der Gewebelehre des Menschen. 6. Anfl. 1889.

12. Langerhans, Ueber Tastkörperchen und Rete Malpighi. Arch. f. mikr. Anat. Bd. 9. 1873.

13. Ledermann, Ueber den Fettgehalt der normalen Haut. Arch. f. Dermatol. und Syphil. Ergänzungrsh. 1892.

14. Liehreich, Ueber das Lamolin etc. Berl. klin. Wochenschrift No. 47. 1885 . 
15. Löwenbach, Sitzungsber. der biolog. Abtheil. des ärtt. Vereins Hamburg. Münch. med. Wochenschr. Nr. 22. 1899.

16. Merk, Experimentelles zur Biologie d. menschl. Haut. 1. Mittheil. Die Beziehungen der Hornschicht zum Gewebesaft. Sitzungsber. der k. Akad. d. Wissensch. Wien. Math.-naturw. KI. Bd. 108. 1899.

17. Derselbe, Ueher den Bau der mensehlichen Hornzelle. Arch. f. mikr. Anat. Bd. 56.1900.

18. Munk, J., Artikel „Fett" in Eulenburg's Realencyclopädie der ges. Heilkunde. 1895.

19. Oehl, Indagini di anatomia microscopica per servire allo studio dell' epidermide e della cute palmare della mano. Annali universali di medicina Bd. 160.1857.

20. Rabl, Untersuchungen über die menschliche Oberhaut und ihre Anhangsgetbilde mit bes. Rücksicht auf die Verhornung. Arch. $f$. mikx. Anat. Bd. 48. 1897.

21. Ranvier, Traité technique d'histologie. 1875.

22. Derselbe, Sur une substance nouvelle de l'épiderme et sur le processus de kératinisation du revêtement épidermique. Compt. rend. de l'Accad. d. scienc. Bd. 88. 1879.

23. Derselbe, De l'éléidine et de la répartition de cette substance dans la peau, la muqueuse buccale et oesophagienne des vertèbrés. Arch. d. physiol. 1884 .

24. Derselbe, Histologie de la peau. Arch. d'anatomie microscopique. Bd. 3. 1899.

25. Ra u sch, Tinctorielle Verschiedenheiten und Relief der Hornzellen. Monatsh. f. pract. Dermatol. Bd. 24. 1897.

26. Sata, Ueber das Vorkommen von Fett in der Haut und in einigen Drüsen, den sog. Eiweissdrüsen. Beiträge z. pathol. Anat. und z. allgem. Pathol. Bd. 27. 1900.

27. Starke, Ueber Fettgranula und eine neue Eigenschaft des Osmiumtetraoxyds. Arch. f. Anat. und Physiol. Phys. Abtheil. 1895.

28. Török, Die Seborrhoea corporis (Duhring) und ihr Verhältniss zur Psoriasis vulgaris und zum Eczem. Arch. f. Dermatol. und Syphil. Bd. 47. 1899.

29. Unna, Beiträge zur Histologie und Entwirklungsgeschichte der menschlichen Oberhaut und ihrer Anhang'sgebilde. Arch. f. mikr. Anat. Bd. 12. 1876.

30. Derselbe, Entwicklungsgeschichte und Anatomie der Haut. Ziemssen's Handbuch der spec. Pathol. und Therapie Bd. 14. 1883.

31. Derselbe, Ueber die Fettfunction der Knäueldrüsen und die Durchsetzung der Haut mit Fett. Verh. d. anatom. Gesellsch. in Kiel. 1898.

32. Derselbe, Der Nachweis des Fettes in der Haut durch secundäre Osmirung. Monatsh. f. pract. Dermatol. Bd. 26. 1898.

33. Weidenreich, Ueber Bau und Verhornung der menschlichen Oberhaut. Arch. f. mikrosk. Anat. Bd. 56. 1900. 
34. Wentscher, Experimentelle Studien über das Eigenleben menschlicher Epidermiszellen ausserhall des Organismus. Beiträge zur patholog. Anat. und z. allgemeinen Pathol. Bd. 24. 1898.

35. Z ander, Untersuchungen über den Verhornungsprocess. II. Mittheilung. Der Bau der menschlichen Epidermis. Arch. f. Anat. u. Physiol. Anat. Abtheil. 1888.

\section{Erklärung zu den Abbildungen auf Tafel XXX u. XXXI.}

(Wo nicht anders bemerkt, entstammt die Haut der Fusssohle des erwachsenen Mensehen; die Zeichnungen sind mit dem A b b e ssehen Zeichenapparate in der Höhe des Objecttisches entworfen.)

Fig. 1. Eleidin im Stratum lucidum. Alkoholhärtung (4 Stunden). Rasirnesserschnitt; Färbung mit Congoroth. Leitz Obj. 7, Oc. 1. $a=$ Kugelform mit Ringbildung; $b=$ ovale Formen.

Fig. 2. Vertheilung des Eleidins im Stratum corneum. Geschnitten und gefuirbt wie Fig. 1. Leitz Obj. 3, Oc. 4. $c=$ Cutis, $s g=$ Strat. germinativ., $s l=$ Strat. lucid., $s r=$ Zone B, $s t=$ Zone C, $s d=$ Zone $\mathrm{D}, e=$ Eleidin.

Fig. 3. Eleidintropfen im Stratum tensum (Zone C). Geschnitten und fixirt wie Fig. 1. Leitz Obj. 7, Oc. 1, $s l=$ Strat. lucid., $s r=$ Zone $\mathrm{B}, s t=$ Zone $\mathrm{C}, s d=$ Zone $\mathrm{D}, e=$ Eleidin.

Fig. 4. Eleidin aus dem Stratum lucidum. Kurze Tanninsäurefixation Celloidineinbettung. Fürbung mit Congoroth. Leitz Obj. 7 Oc. 3. $c=$ Kernhöhle, $e=$ Eleidin.

Fig. 5. Zelleu des Stratum corneum nach lingerer Tanninsäurefixation. Celloidineinbettung. Färbung mit Hämalaun und Congoroth. Leitz Obj. 7, Oc. $1 . \quad s g r=$ Strat. granulos., $s l=$ Strat. lucid., $s r=$ Zone B, $m=$ Zellmembran, $f=$ Fasernetz, $c=$ Kernhöhle, $n=$ geschrumpfte Kerne.

Fig. 6. Zellen des Stratum corneum bei starker Vergrösserung. Behandlung wie eben. Härbung mit Congoroth. Ze i ss Ap. 2 mm, Oc. 6. $f=$ Fasernetz, $c=$ Kernhöhle, $n=$ geschrumpfter Kern.

Fig. 7. Epidermis nach Unna fixirt. Celloidineinbettung. Färbung: mit Congoroth. Leitz Obj. 3, Oc. 1. $p=$ Cutispapille, $s g=$ Strat. germinat., $s l=$ Strat lucid., $s r=$ Zone $B$, $s t=$ Zone C, $s d=$ Zone $\mathrm{D}, p i=\mathrm{B}$ laschko'sche Falte.

Fig. 8. Dasselbe Präparat, secundär osmirt. Leitz Obj. 3, Ocul. 4. Buchstabenbezeichnung die gleiche wie von Fig. 7.

Fig. 9. Schena eines Schnittes durch die Epidermis von Vola manus oder Planta pedis, senkrecht zu den Leisten der Oberfläche (vgl. Text Seite 602 u. f.). $p a=$ Pars arcuata (Drüsenleistentheil), 
$p i=$ Pars implicita (Blaschko'sche Falte), $s g=$ Strat. germinativ., $s g r=$ Stratum granulos., $s l=$ Strat. lucid. (Zone A), $s r=$ Stratum relaxat. (Zone B), st = Strat. tensum (Zone C), $s d=$ Strat. disjunct. (Zone D), vs = Capillarschlingen der Cutispapillen, $d s=$ Ausfülırgang einer Schweissdrüse.

Fig. 10. Verhalten des Stratum lucidum bei Osmiumfixation. Zwölfstündige Osmiumhärtung; Paraffineinbettung. Saffraninfärbung. Leitz Obj. 7, Oc. 1. $s g r=$ Strat. granulos., $s l=$ Strat. lucid., $b=$ basaler Osmiunstreifen, $i=$ noch nicht osmirte Hornschicht.

Fig. 11. Eleidinkugel in einer Zelle des Stratum granulosum. Haut der Fingerbeere. Osmiumfixation (24 Stunden); Paraffineinbettung. Z eis s Ap. $2 \mathrm{~mm}$, Oc. $6 . \quad n=$ Kern, $k=$ Keratohyalingranula $e=$ Eleidintropfen.

Fig. 12. Angebliche Fettkörnchen in dem Stratum germinativum. Fixation, Einbettung und Osmirung nach Unna. Leitz Obj. 7, Oc. 1. $c=$ Cutis, $s g=$ Strat. germinat., $g=$ schwarze Körnchen fraglicher Natur.

Fig. 13. Zellen des Stratum disjunctum. Osmiumfixirung (24 Stunden). Paraffineinbettung. Leitz Obj. 7, Oc. 1. $f=$ Netzwerk im Zellinnern, $c=$ Kernhöhle.

Fig. 14. Uebergang des Stratum lucidum in das Stratum relaxatum. Siebenstündige Osmiumfixirung. Paraffineinbettung. Z e is s Ap. $2 \mathrm{~mm}$, Oc. $4 . \quad s l=$ Strat. lucid., $s r=$ Strat. relaxat., $i s=$ Intercellularräume.

Fig. 15. Basaler Theil des Stratum corneum. Fixation in Alkohol, secundäre Osmirung. Leitz Obj. 7, Oc. 1. sgr $=$ Strat. granulos., sc. $=$ Strat. corn., is = Intercellularräume.

\title{
Ganglienzellen in der Schlundmusculatur von Pulmonaten.
}

\author{
Von
}

\section{Dr. H. Smidt.}

Hierzu Tafel XXXII.

Nacbdem schon seit mehr als 30 Jahren die Ganglienzellen des Herzens der Wirbelthiere bekannt und vielfach untersucht waren, erschien 1876 eine Arbeit von Dogiel "uber die Anatomie 